\title{
Circuits that encode and predict alcohol associated preference
}

\author{
Kristin M. Scaplen ${ }^{\mathrm{a}}$, Mustafa Talay ${ }^{\mathrm{b}}$, Sarah Salamon ${ }^{\mathrm{c}}$, Kavin M. Nuñez ${ }^{\mathrm{d}}$, Amanda G. Watermana ${ }^{\mathrm{a}}$, Sydney \\ Gang $^{\mathrm{e}}$, Sophia L. Song ${ }^{\mathrm{a}}$, Gilad Barnea ${ }^{\mathrm{a}}$, Karla R. Kaun ${ }^{\mathrm{a}, 1}$ \\ a Department of Neuroscience, Brown University, Providence, RI 02912, USA \\ ${ }^{\mathrm{b}}$ Howard Hughes Medical Institute, Department of Molecular and Cellular Biology, Harvard University, Cambridge, MA 02138, \\ USA \\ ${ }^{c}$ Department of Pharmacology, University of Cologne, Faculty of Medical and University Hospital Cologne, Germany \\ ${ }^{\mathrm{d}}$ Department of Molecular Pharmacology and Physiology, Brown University, Providence, RI 02912, USA \\ ${ }^{e}$ Department of Biochemistry, Brown University, Providence, RI 02912, USA \\ ${ }^{1}$ Corresponding author: karla_kaun@brown.edu
}

\begin{abstract}
Substance use disorders are chronic relapsing disorders often impelled by enduring memories and persistent cravings. Alcohol, as well as other addictive substances, remolds neural circuits important for memory to establish obstinate preference despite aversive consequences. How pertinent circuits are selected and shaped to result in these unchanging, inflexible memories is unclear. Using neurogenetic tools available in Drosophila melanogaster we define how circuits required for alcohol associated preference shift from population level dopaminergic activation to select dopamine neurons that predict behavioral choice. During memory expression, these dopamine neurons directly, and indirectly via the mushroom body (MB), modulate the activity of interconnected glutamatergic and cholinergic output neurons. Transsynaptic tracing of these output neurons revealed at least two regions of convergence: 1) a center of memory consolidation within the MB implicated in arousal, and 2) a structure outside the MB implicated in integration of naïve and learned responses. These findings provide a circuit framework through which dopamine neuron activation shifts from reward delivery to cue onset, and provides insight into the inflexible, maladaptive nature of alcohol associated memories.
\end{abstract}

Keywords: Mushroom Body, Alcohol, Memory, Addiction, Dopamine

\section{Introduction}

An organism's behavior is guided by memories of past experiences and their associated positive or negative outcomes. The accuracy of these associations is vital to the organism's survival. In a changing environment, however, successful associations should be dynamic and malleable, providing opportunities for updating associations based on new information. In substance use disorders (SUD) this flexibility is often absent or difficult to achieve (Font and Cunningham 2012, Torregrossa and Taylor 2013). Preference and cravings for drugs of abuse, including alcohol, persist in the face of aversive consequences leading to maladaptive drug seeking behaviors and a devastating economic and social impact on individuals, communities, and society as a whole. Understanding the circuitry mechanisms that underlie how these memories are encoded and retrieved is critical to understanding why these memories are so resistant to change.

Systems memory consolidation suggests that individual brain regions have a time limited role in the recall of memories as they are slowly reorganized and transferred to anatomically distinct regions in the brain. More recent work has suggested that even within a brain region, such as the hippocampal formation, circuits important for encoding and retrieval are distinct (Roy, Kitamura et al. 2017, Cembrowski, Phillips et al. 2018). Strikingly, drugs of abuse, such as alcohol disrupt these memory systems resulting in enduring preferences, attentional bias for associated cues (Field and Cox 2008, Fadardi, Cox et al. 2016), and habitual behaviors. The neuronal genetic, morphologic, and physiologic diversity that exists within the brain, however, has precluded mammalian animal models from accessing the level of spatial resolution in a temporally specific manner required to identify and investigate microcircuits important for alcohol associated behaviors. We sought to address how drugs of abuse can shift memory from being flexible to inflexible by using a combination of behavioral, thermogenetic, in vivo calcium imaging, and transsynaptic tracing techniques in Drosophila melanogaster.

The neural circuitry underlying the Drosophila reward response is complex and remarkably similar to mammals (Scaplen and Kaun 2016). Drosophila exhibit sophisticated behaviors in response to changes in their environment, including formation of appetitive memories for food associated with environmental cues (Waddell 2010, Kahsai and Zars 2011, Schleyer, Saumweber et al. 2011, Herrero 2012, Fernandez and Kravitz 2013, Heisenberg 2015, Owald, Lin et al. 2015). Drosophila also form appetitive memories for the pharmacological properties of alcohol (Kaun, Azanchi et al. 2011, Nuñez, Azanchi et al. 2018). These alcohol memories are persistent and impel flies to walk over a $120 \mathrm{~V}$ electric shock in the presence of associated cues. This type of goal directed behavior, in the face of aversive consequences, suggest that alcohol associated memories are more inflexible than memories for natural reward like sucrose (Kaun et al, 2011). Drosophila provides an ideal model to investigate these differences as the neurogenetic tools available in this species permits dissection of memory circuits with unprecedented temporal and spatial resolution. 
Scaplen et al., 15 Mar 2019 - preprint copy - BioRxiv

In Drosophila the establishment of alcohol preference requires an associative central brain structure called the mushroom bodies (MB) and dopamine (DA) (Kaun, Azanchi et al. 2011). We show that circuits required for formation of alcohol preference shift from population-level dopaminergic encoding to two microcircuits comprising of interconnected dopaminergic, glutamatergic, and cholinergic neurons. These circuits converge onto the fan-shaped body (FSB), a higherorder brain center implicated in arousal and modulating behavioral response (Liu, Seiler et al. 2006, Weir, Schnell et al. 2014, Weir and Dickinson 2015, Pimentel, Donlea et al. 2016, Qian, Cao et al. 2017, Donlea, Pimentel et al. 2018, Hu, Peng et al. 2018, Troup, Yap et al. 2018). Our results, therefore, provide an in vivo circuit framework for how drugs of abuse temporally regulate acquisition and expression of sensory memories, which ultimately results in a shift in behavioral response from malleable to inflexible.

\section{Results}

\section{Dopamine neurons innervating the mushroom body are required for} alcohol reward associations

Dopamine has a long-standing role in addiction and a defined role in reward-related behavioral learning that spans across species (Yoshimoto, McBride et al. 1992, Robbins and Everitt 2002, Hyman, Malenka et al. 2006, Wanat, Willuhn et al. 2009, Kaun, Azanchi et al. 2011, Torregrossa, Corlett et al. 2011, Scaplen and Kaun 2016). In mammals, midbrain ventral tegmental area (VTA) and substantia nigra pars compacta $(\mathrm{SNc})$ structures are primary sources of dopamine responsible for modulating synaptic plasticity within the brain. However, the neuronal heterogeneity that exists within these structures, and lack of necessary spatial and temporal resolution to parse apart subpopulations or isolate individual neurons in behaving animals, have precluded the field from gaining a complete understanding of how dopaminergic neurons engage and augment underlying neural activity both in normal learning and addiction.

We first sought to identify which dopaminergic neurons within the Drosophila brain are necessary for alcohol associated preference. In Drosophila a discrete population of protocerebral anterior medial (PAM) dopamine neurons have an identified role in detecting and processing natural rewards. PAM neurons are required for the acquisition of sucrose memory (Liu, Placais et al. 2012, Huetteroth, Perisse et al. 2015, Yamagata, Ichinose et al. 2015), and known to be activated by sucrose administration (Liu, Placais et al. 2012, Harris, Kallman et al. 2015). We first tested the requirement of activity of PAM dopamine neurons in alcohol associative preference (Figure 1a). For selective manipulations of these neurons, we expressed the dominant negative temperature sensitive shibire $\left(s h i^{t s l}\right)$ using R58E02-GAL4 (Figure 1b). To establish temporal requirements, we temporarily and reversibly inactivated neurotransmission by raising the temperature to restricted levels $\left(30^{\circ} \mathrm{C}\right)$ during acquisition, the overnight consolidation period, or retrieval (Figure 1a). Acquisition was defined as the time during which an odor was presented in isolation (unpaired odor) and a second odor was paired with an intoxicating dose of vaporized ethanol (paired odor). During acquisition, reciprocally trained flies received three of these spaced cue (odor) and overlapping cue sessions (odor + ethanol). Post-acquisition, flies were given a choice 24 hours later between the unpaired and paired odor in a $\mathrm{Y}$ maze (Figure 1a). Retrieval was defined as the time during which the flies chose between the unpaired and paired odors 24 hours post acquisition.

Previous work established that flies show preference for the cues associated with ethanol intoxication 24 hour later, regardless of odor identity (Kaun et al 2011). We found inactivating neurotransmission in PAM dopaminergic neurons during acquisition or retrieval, but not during the overnight consolidation, significantly reduced preference for cues associated with ethanol (Figure 1c). Strikingly, despite dopamine's established role in modulating locomotor and motor responses (Romo and Schultz 1990, Lima and Miesenbock 2005, Schultz 2007, Dodson, Dreyer et al. 2016, Howe and Dombeck 2016, Syed, Grima et al. 2016, da Silva, Tecuapetla et al. 2018), inactivating PAM dopaminergic neurons did not affect ethanol induced activity (Supplementary Figure 1). Together, these results suggest that neurons required for encoding preference are not required for the locomotor response to the acute stimulatory properties of ethanol. Formation of alcohol memories, instead, occurs within the circuit framework defined by memories for natural reward.

\section{Dopaminergic encoding of alcohol memory acquisition occurs at the population level}

To determine how alcohol influenced activity of PAM dopaminergic neurons to shift memories from malleable to inflexible, we first used a dopamine staining protocol to label dopamine within the brain following 10 minutes of air or alcohol. As expected there was a significant amount of dopamine labeled within the mushroom body and the majority of fluorescence was limited to the horizontal lobes (Figure 1d). We hypothesized that dopamine fluorescence would increase within the horizontal lobes of the mushroom body in response to alcohol, however, quantification of fluorescence did not provide significant differences (Supplementary Figure 2). We reasoned that dopamine staining likely could not distinguish between dopamine in the presynaptic terminals and dopamine in the synaptic cleft, and thus turned to 2-photon functional calcium imaging to monitor circuitry dynamics of PAM dopaminergic activity in the context of intoxicating alcohol. We used R58E02-Gal4 to express GCaMP6m (Chen, Wardill et al. 2013) and recorded from the PAM presynaptic terminals at the MB while naïve flies were presented with 10 minutes of odor, followed by 10 minutes of odor plus intoxicating doses of alcohol (Figure 1e).

Interestingly, early in the respective recording sessions (odor vs odor + alcohol), changes in calcium dynamics was greater in the odor only group (Figure 1e, 1f), however with prolonged alcohol exposure, greater calcium dynamics started to emerge from the population of dopamine neurons in the odor + alcohol group (Figure 1f). This suggests that the pharmacological properties of alcohol increase dopaminergic neuronal activity in vivo, nicely complementing in vitro physiology in VTA slices that demonstrate VTA activity is increased by moderate alcohol doses (Brodie and Appel 1998, Morikawa and Morrisett 2010) and micro dialysis studies that report increased dopamine in the nucleus accumbens (Di Chiara and Imperato 1985, Imperato and Di Chiara 1986, Yoshimoto, McBride et al. 1992, Di Chiara, Tanda et al. 1996, Bassareo, De Luca et al. 2003, Howard, Schier et al. 2008), central amygdala (Yoshimoto, Ueda et al. 2000), and medial prefrontal cortex (Ding, Oster et al. 2011). However, the complexity of mammalian VTA circuits has obscured our understanding of whether increased dopamine release in downstream regions is due to more firing of discrete populations of VTA dopamine neurons, or recruitment of the larger population of VTA dopamine neurons.

To address whether specific subsets of dopamine neurons within the PAM neuron population are necessary for alcohol associated preference, we blocked transmission in subsets of these neurons using 18 highly specific split-Gal4 lines during both acquisition and retrieval. We found that preference was disrupted when neurotransmission was blocked in DA neurons projecting to the medial aspect of horizontal 
bioRxiv preprint doi: https://doi.org/10.1101/578401; this version posted March 15, 2019. The copyright holder for this preprint (which was not certified by peer review) is the author/funder, who has granted bioRxiv a license to display the preprint in perpetuity. It is made available under aCC-BY-ND 4.0 International license.

Scaplen et al., 15 Mar 2019 - preprint copy - BioRxiv

MB (Supplementary Figure 3). We therefore selected split-Gal4 lines that targeted the medial aspect of the horizontal lobe and determined their role specifically in acquisition of alcohol associated preference. Surprisingly, unlike long-term sucrose memory (Ichinose, Aso et al. 2015, Yamagata, Ichinose et al. 2015), thermogenetic inactivation of specific subsets of dopamine neurons innervating compartments of the medial horizontal lobe during acquisition did not disrupt 24-hour alcohol associated preference (Figure 2a-h, Supplementary Figure 4a). Cell counts of the broadest split-GAL4 lines (40B and 42B), HL9 and a
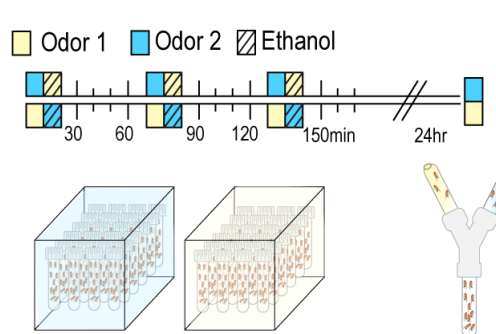

Group 1

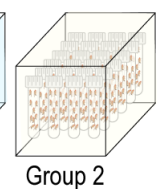

Conditioned $=$ \# flies paired odor - \# flies unpaired odor Preference
Index

d

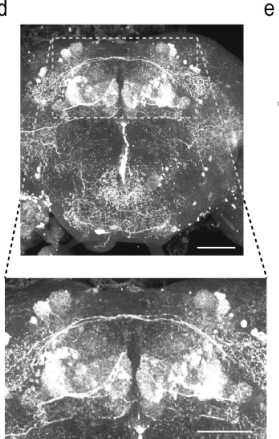

,

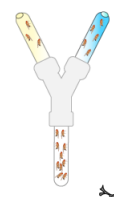

$+\frac{1}{2+2}$ total \# flies
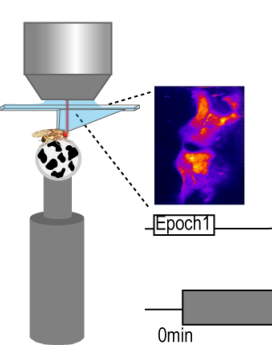

Epoch1
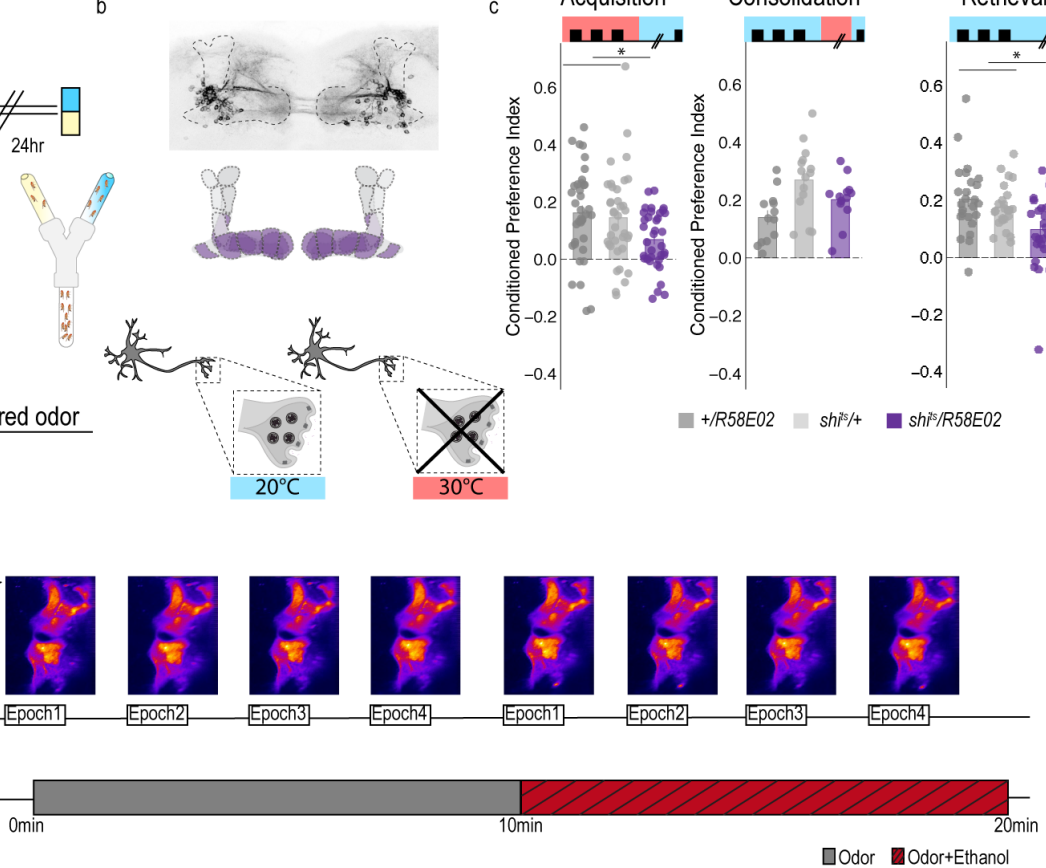

Epoch 1
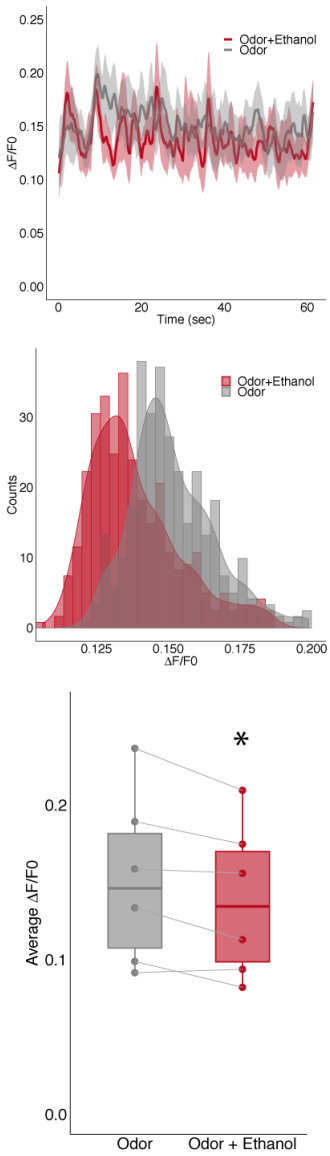

Epoch 2
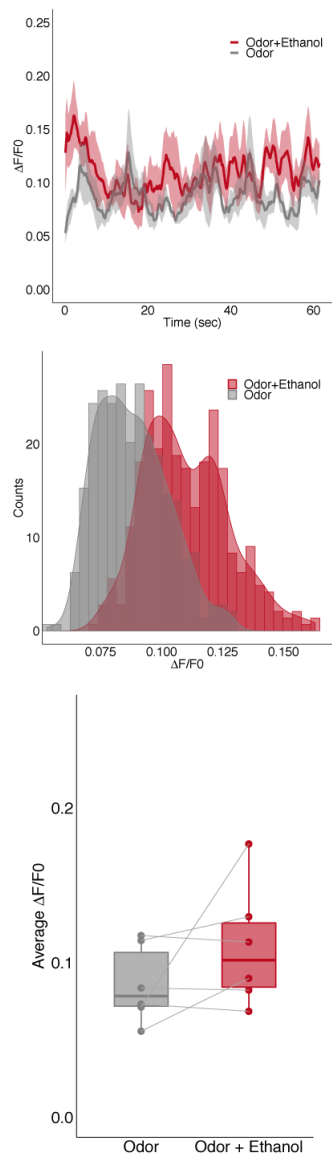

Epoch 3
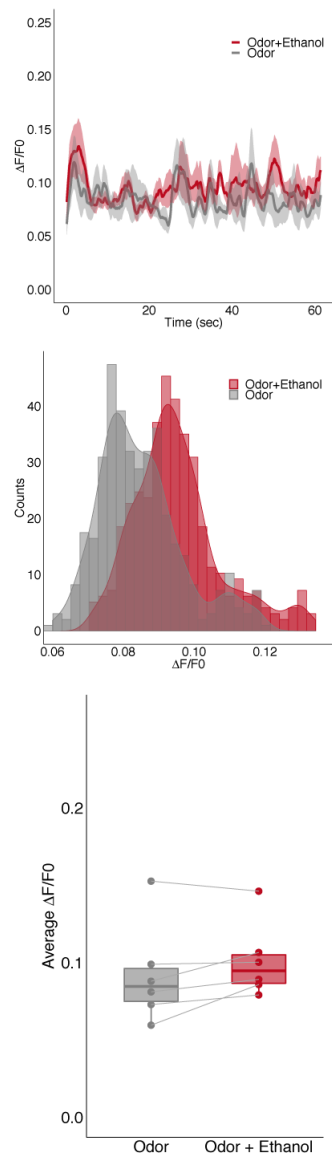

Epoch 4
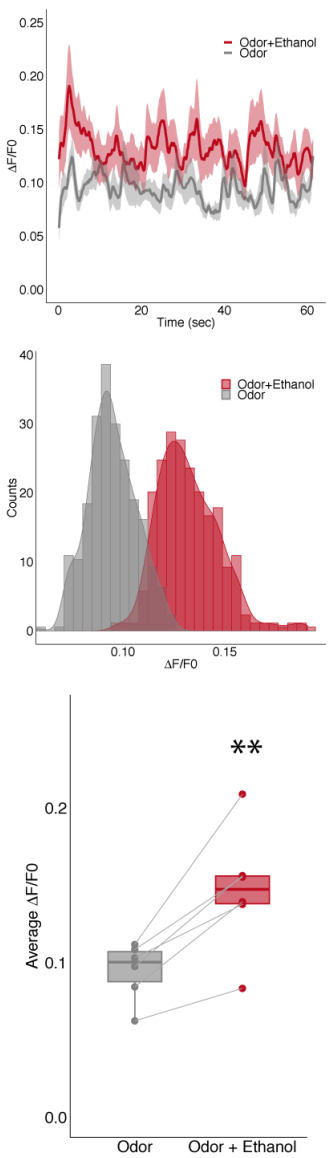

\section{Retrieval}

ann

0.6

$-0.5$

$-0.4$

shis/R58E02

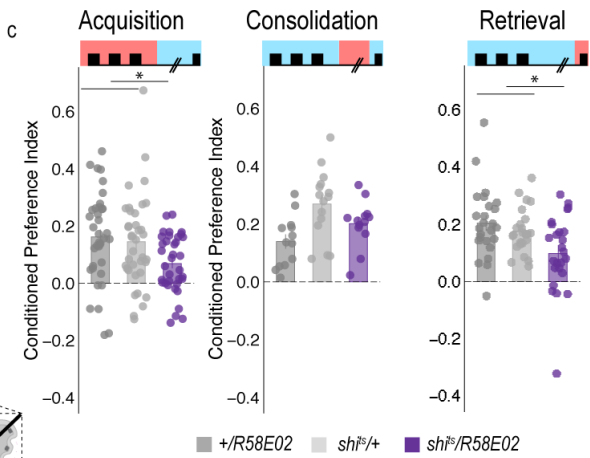


bioRxiv preprint doi: https://doi.org/10.1101/578401; this version posted March 15, 2019. The copyright holder for this preprint (which was not certified by peer review) is the author/funder, who has granted bioRxiv a license to display the preprint in perpetuity. It is made available under aCC-BY-ND 4.0 International license.

Scaplen et al., 15 Mar 2019 - preprint copy - BioRxiv

Fig. 1. PAM dopaminergic neurons are necessary for encoding alcohol associated preference. (a) Schematic illustrating odor condition preference paradigm. Vials of 30 flies are presented with three sessions of 10 minutes of an unpaired odor, followed by 10 minutes of a paired odor plus intoxicating vaporized ethanol. To control for odor identity, reciprocal controls were used. (b) PAM dopaminergic neurons innervate the horizontal lobe of MB. Confocal stack illustrates innervation pattern using R58E02>MCD-GFP. Dashed line outlines MB region. MB schematic illustrates MB compartments innervated by PAM neurons. Lower schematic highlights mechanisms of thermogenetics using shibire ${ }^{\text {ts } 1}$. Temperature is used to inactivate neurotransmission at restrictive temperatures $\left(30^{\circ} \mathrm{C}\right)$, but not at permissive temperatures $\left(20^{\circ} \mathrm{C}\right)$. (c) PAM dopaminergic neurons activity is necessary during acquisition and retrieval, but not consolidation. Bar graphs illustrate mean +/- standard error of the mean. Raw data are overlaid on bar graphs. Each dot is an $\mathrm{n}$ of 1 , which equals approximately 60 flies (30 per odor pairing). One-way ANOVA with Tukey Posthoc was used to compare mean and variance. ${ }^{*} p<0.05$ (d) Example confocal stack labeling dopamine within the brain. Dopamine labeling is concentrated around the MB. (e) Schematic of calcium imaging protocol. Flies are exposed to odor followed by odor plus intoxicating vaporized ethanol while resting or walking on a ball. We used the same odor for both conditions so we could better compare circuit dynamics in response to ethanol and control for odor identity. Fluorescence was captured for $61 \mathrm{sec}$ recording epochs that were equally spaced by 2 minutes. Example maximum intensity fluorescent stacks are shown for each epoch. (f) Average traces recorded during the odor and odor plus ethanol epochs. Middle panels illustrate the binned $\Delta \mathrm{F} / \mathrm{F} 0$ and highlights a change in calcium dynamics as a consequence of ethanol exposure. Lower panels illustrate the average $\Delta F / F 0$ for each fly in each condition at each epoch. Within Subject Repeated Measures ANOVA with a Bonferroni Posthoc was used to compare mean and variance across condition and time. Scale bar $=50 \mu \mathrm{m}{ }^{*} p<0.05$ ${ }^{* *} \mathrm{p}<0.01$

R58E02 driver lines revealed that despite targeting nearly all of the horizontal lobes of the MB, 40B, 42B, and HL9 expressed in significantly fewer cells (Supplementary Table 1). Together these data suggest that unlike long-term sucrose memory, subsets of dopamine neurons are not responsible for the acquisition of alcohol associated preference, and disruption of this process requires the disruption of population level dopaminergic activity. Thus, we speculate that alcohol memories are enduring and less adaptive than sucrose memories because alcohol engages a larger number of dopamine neurons during memory acquisition (Figure 2i).

\section{Memory expression is dependent on a sparse subset of dopamine neurons}

A hallmark of reward-encoding dopamine neurons is the gradual transfer of neural activation from reward delivery to cue onset during associative learning. During learning, dopaminergic activity shifts from responding to a reward during acquisition to the cue that predicts a reward during expression of the associated memory (Keiflin and Janak 2015, Schultz 2015, Schultz 2016). However, the circuit mechanisms underlying this shift and knowledge about whether all dopamine neurons do this, or whether a selective subset of dopamine neurons respond to the cue are unknown. It's critical to understand this if we want to understand how drugs of abuse (alcohol) influence these circuits to result in such long-lasting, inflexible memories.

We therefore used a thermogenetic approach, as previously described, to temporarily inactivate neurotransmission in subsets of dopamine input neurons during retrieval, but not during acquisition or consolidation using a set of highly specific split-Gal4 lines. Strikingly, only inactivating dopaminergic neurons innervating $\beta$ 'a compartment of the MB, using split-Gal4 line 109B, significantly reduced alcohol associated preference suggesting that these neurons are important for the expression of alcohol associated preference during retrieval (Figure 3 ). This suggests population encoding during acquisition shifts to sparse representation during memory expression.

\section{A dopamine-glutamate circuit regulates memory expression}

Systems memory consolidation suggests that there are different circuits for memory acquisition and expression. Indeed, work in both fly and mammalian models suggest brain regions have a time limited role in systems consolidation. For example, recent work shows evidence of distinct acquisition and retrieval hippocampal circuits for contextually based fear memory (Roy et al 2017). Our next goal was to map the circuits through which the dopamine signal during retrieval drives the behavioral decision to move towards the paired cue.
To determine the circuit mechanisms that regulate a shift from dopaminergic population encoding during acquisition to sparse representation during retrieval, we tested the requirement of $\mathrm{MB}$ output neurons (MBONs) that aligned with the $\beta$ ' $2 \mathrm{a}$ dopamine neurons required for memory expression. Inactivating the output of glutamatergic MBONs innervating similar compartments during acquisition, using 4 different split-Gal4 lines, did not significantly reduce alcohol associated preference (Figure 4A-D). However, similar inactivation during retrieval identified a single $\beta 2 \beta^{\prime} 2$ a glutamatergic MBON important for the expression of alcohol associated preference (Figure 4E-H).

Thus far, we have defined a putative microcircuit that consists of a single subset of 8-10 dopamine neurons innervating the $\beta^{\prime} 2 \mathrm{a} \mathrm{MB}$ compartment and a single glutamatergic MBON that also innervates the $\beta^{`} 2 \mathrm{a} \mathrm{MB}$ compartment ( $\left.\beta 2 \beta^{`} 2 \mathrm{a}\right)$ that are important for the expression of alcohol associated preference (Figure $4 \mathrm{~m}$ ). Previous work suggested that $\beta$ 2a dopaminergic neurons were anatomically connected with $\beta$ '2amp MBONs at the level of the MB, however, it was unclear which MBON $\beta$ '2a dopaminergic neurons were synaptically connected (Lewis et al 2015). To confirm connectivity between $\beta$ ' 2 a dopaminergic neurons and $\beta 2 \beta^{\prime} 2 \mathrm{a}$ MBONs we used the recently developed anterograde transsynaptic labeling method trans-Tango to label the postsynaptic targets of the $\beta$ ' 2 a dopaminergic neurons (Talay, Richman et al. 2017 Figure 5a). Crossing split-Gal4 line $M B 109 B$ with transTango flies revealed $\alpha^{\prime} \beta^{\prime}$ MB neurons as postsynaptic to $\beta^{\prime} 2 \mathrm{a}$ dopaminergic neurons (Figure 5a). Interestingly, $\beta{ }^{`} 2 \mathrm{mp} \mathrm{MBON}$, and not $\beta 2 \beta^{\prime} 2 \mathrm{a}$ MBON were labeled as post synaptic to $\beta^{`} 2 \mathrm{a}$ dopaminergic neurons suggesting that connections between $\beta^{\prime} 2 \mathrm{a}$ dopaminergic neurons and $\beta 2 \beta^{\prime} 2 \mathrm{a}$ glutamatergic MBONs are not direct, but likely interact via intrinsic cholinergic MB neurons (Barnstedt, Owald et al. 2016).

To exclude the possibility of a false negative result, we confirmed the lack of functional connectivity between dopaminergic neurons and $\beta 2 \beta^{`} 2 \mathrm{a}$ using dopamine receptor RNAi (Supplementary Figure 5). RNAi against D1-like receptors or D2Rs in $\beta 2 \beta^{\prime} 2$ a neurons did not disrupt alcohol associated preference (Figure $4 \mathrm{j}-1$ ). Combined, these data suggest the functional connectivity between $\beta^{\prime} 2 \mathrm{a}$ dopaminergic neurons and the $\beta 2 \beta$ ' $2 \mathrm{a}$ is not required for alcohol associated preference, but that expression of alcohol memories is occurring through a circuit consisting of MB cholinergic neurons, $\beta$ '2a dopamine neurons and $\beta 2 \beta$ ' 2 a glutamate output neurons. Indeed, previous work from our lab reported the requirement of D2Rs in MB neurons for alcohol associated preference (Petruccelli, Feyder et al. 2018). A similar 
Scaplen et al., 15 Mar 2019 - preprint copy - BioRxiv

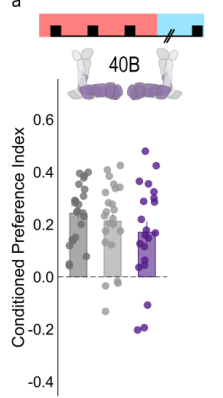

e

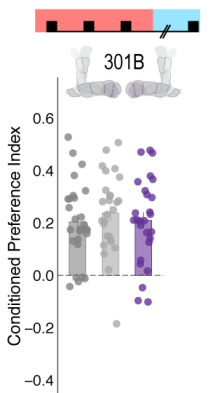

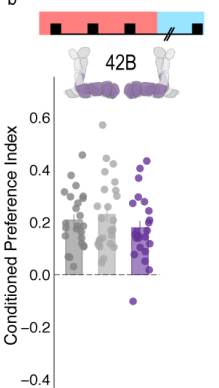
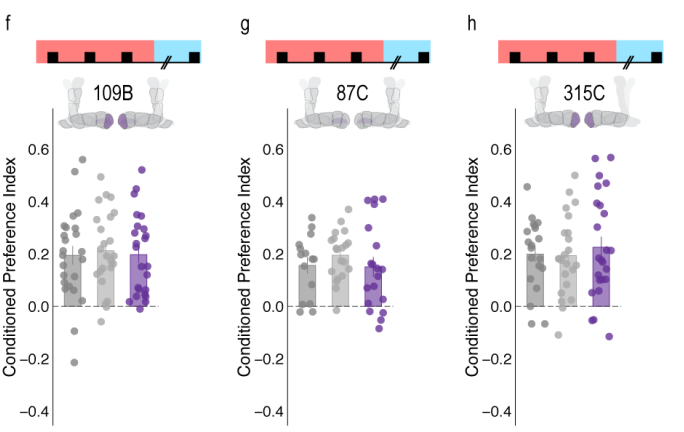

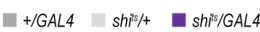
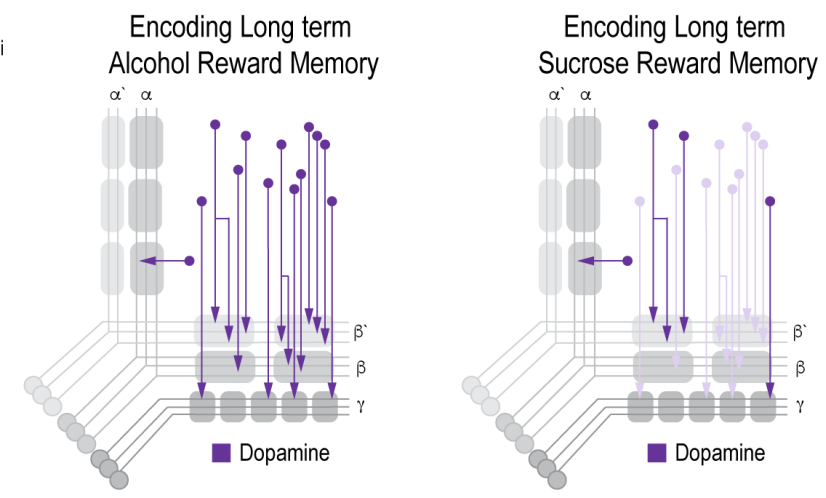

Fig. 2. Subsets of PAM dopaminergic neurons are dispensable for encoding alcohol associated preference. (a-h) Using thermogenetics to inactivate neurotransmission during acquisition in dopaminergic neurons with varying expression patterns did not disrupt alcohol associated preference. Split-Gal4 lines tested are ordered by MB innervation patterns. Bar graphs illustrate mean $+/$ - standard error of the mean. Raw data are overlaid on bar graphs. Each dot is an $n$ of 1, which equals approximately 60 flies (30 per odor pairing). One-way ANOVA was used to compare mean and variance. (i) Model comparing encoding of alcohol reward memory and sucrose reward memory. Encoding alcohol reward memory requires population level PAM dopaminergic activity whereas sucrose reward memory is supported by subsets of PAM dopaminergic neurons during acquisition (Ichinose, Aso et al. 2015, Yamagata, Ichinose et al. 2015). The number of dopamine neurons recruited during acquisition may underlie why alcohol reward memories are persistent.

circuit motif was recently described in mice where dopamine regulated the release of acetylcholine from striatal tonically active interneurons on to glutamatergic neurons resulting in chronic decreases in corticostriatal activity during amphetamine withdrawal (Wang, Darvas et al. 2013). This suggests our findings describe a general circuit motif for exploring circuitry mechanisms of reward and motivation.

\section{A separate dopamine-glutamate circuit regulates memory consolidation}

Our transsynaptic tracing method suggests a putative direct synaptic connection between $\beta$ '2a dopamine neurons and $\beta$ '2 $\mathrm{mp}$ glutamatergic output neurons in regulating alcohol associated preference. We functionally validated the connection between $\beta$ '2a dopamine neurons and $\beta^{\prime} 2 \mathrm{mp}$ glutamatergic neurons using dopamine receptor RNAi lines (Figure 5b). Interestingly, decreasing levels of D2R, but not D1Rs, reduced alcohol associated preference (Fig 5b), suggesting a D2Rdependent pathway in MB that regulates alcohol memory.

Similar circuit motifs whereby dopamine directly synapses on glutamatergic neurons in the prefrontal cortex (PFC) have been reported in mammals and importantly, are altered by alcohol (TranthamDavidson and Chandler 2015). These connections might support memory flexibility; however, it is unclear the mechanism by which this works. We hypothesized that neurons important for memory consolidation in our Drosophila MB circuit might modulate expression of memory, thereby influencing flexibility of memory. Previous work in Drosophila reported that activating $\beta 2 \mathrm{mp}$ glutamatergic neurons promoted arousal (Sitaraman et al 2015). Thus, we reasoned that inactivating these neurons while flies normally sleep would further decrease arousal and facilitate memory consolidation. To confirm this hypothesis, we tested how activity of MB074C neurons affected alcohol memory as this line, which expresses highly in $\beta^{`} 2 \mathrm{mp}$, as well as $\beta 2 \beta^{\prime} 2 \mathrm{a}$, and to a much less extent, $\gamma^{5} \beta^{\prime} 2 \mathrm{a}$ (Aso, Hattori et al. 2014) aligns with the predicted $\beta^{\prime} 2 \mathrm{mp}$ postsynaptic cells. Inactivating these subsets of glutamatergic neurons during acquisition or retrieval did not disrupt alcohol associated preference (Figure 5c, d). However, when these neurons were inactivated during the overnight consolidation, alcohol associated preference was enhanced relative to controls (Figure 5e). Together these data suggest that dopamine via $\beta ` 2$ a neurons inhibit the $\beta 2 \mathrm{mp}$ glutamatergic neuron via $\mathrm{D} 2 \mathrm{R}$ receptors which leads to the expression of alcohol associated preference. In the absence of dopamine (Figure 3f) or D2R receptors (Figure 5b), preference is disrupted.

\section{Convergent microcircuits encode alcohol reward expression}

The central role for the $\beta^{\prime} 2 \mathrm{mp}$ in consolidation and memory malleability suggests that this region may integrate information from several circuits required for memory expression. Indeed, there is a wealth of examples in the literature of the systems balancing input from integrating neural circuits to drive goal directed behavior (Knudsen 2007, Buschman and Miller 2014, Hoke, Hebets et al. 2017). Previous anatomical studies predicted that $\beta^{\prime} 2 \mathrm{mp}$ glutamatergic MBON and $\alpha^{\prime} 2$ cholinergic MBON were synaptically connected (Aso, Hattori et al. 2014). trans-Tango confirmed that the $\beta^{`} 2 \mathrm{mp}$ MBON is a postsynaptic target of the $\alpha^{\prime} 2 \mathrm{MBON}$ (Figure 6a).

We previously showed that inactivating the $\alpha^{\prime} 2$ cholinergic output throughout both memory acquisition and expression decreased alcohol associated preference (Aso, Sitaraman et al. 2014). To establish the specific temporal requirements of $\alpha ` 2 \mathrm{MBON}$ and determine whether its corresponding $\alpha 2 \alpha ` 2$ dopaminergic input is necessary for alcohol associated preference, we thermogenetically inactivated neurotransmission during either acquisition or retrieval. Inactivating $\alpha 2$ cholinergic MBONs or its corresponding $\alpha 2 \alpha^{\prime} 2$ dopaminergic neurons during expression, but not acquisition of alcohol memory, significantly reduced alcohol associated preference (Figure 6b-e). The involvement of $\alpha 2 \alpha^{\prime} 2$ dopaminergic neurons is particularly interesting because it part of a separate population of dopamine 
bioRxiv preprint doi: https://doi.org/10.1101/578401; this version posted March 15, 2019. The copyright holder for this preprint (which was not

a
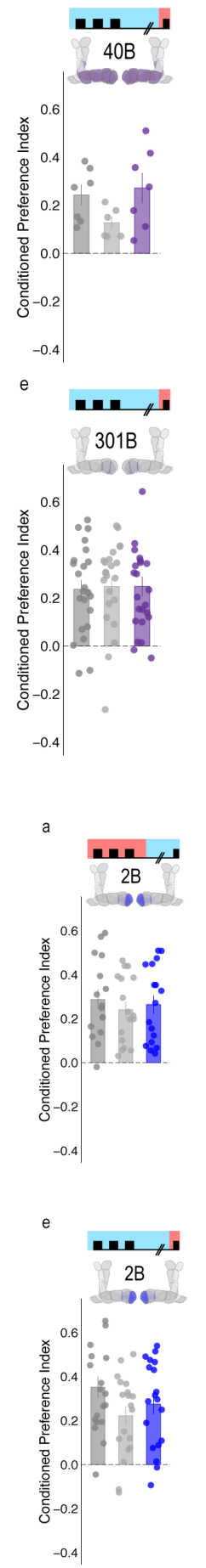
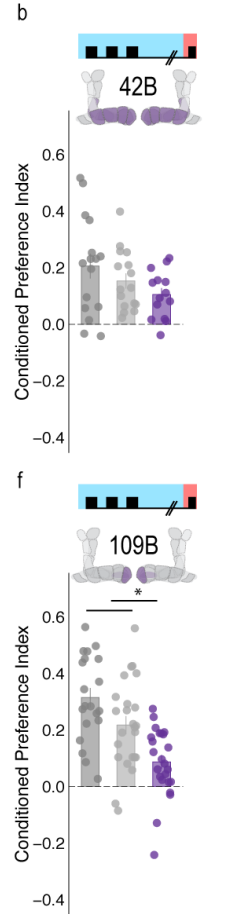
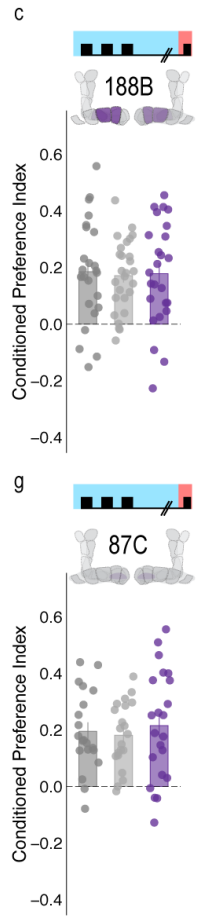

$+/$ GAL4 $\square \operatorname{shis}^{\mathrm{s} / \mathrm{H}} \square \operatorname{shis/GAL4}$
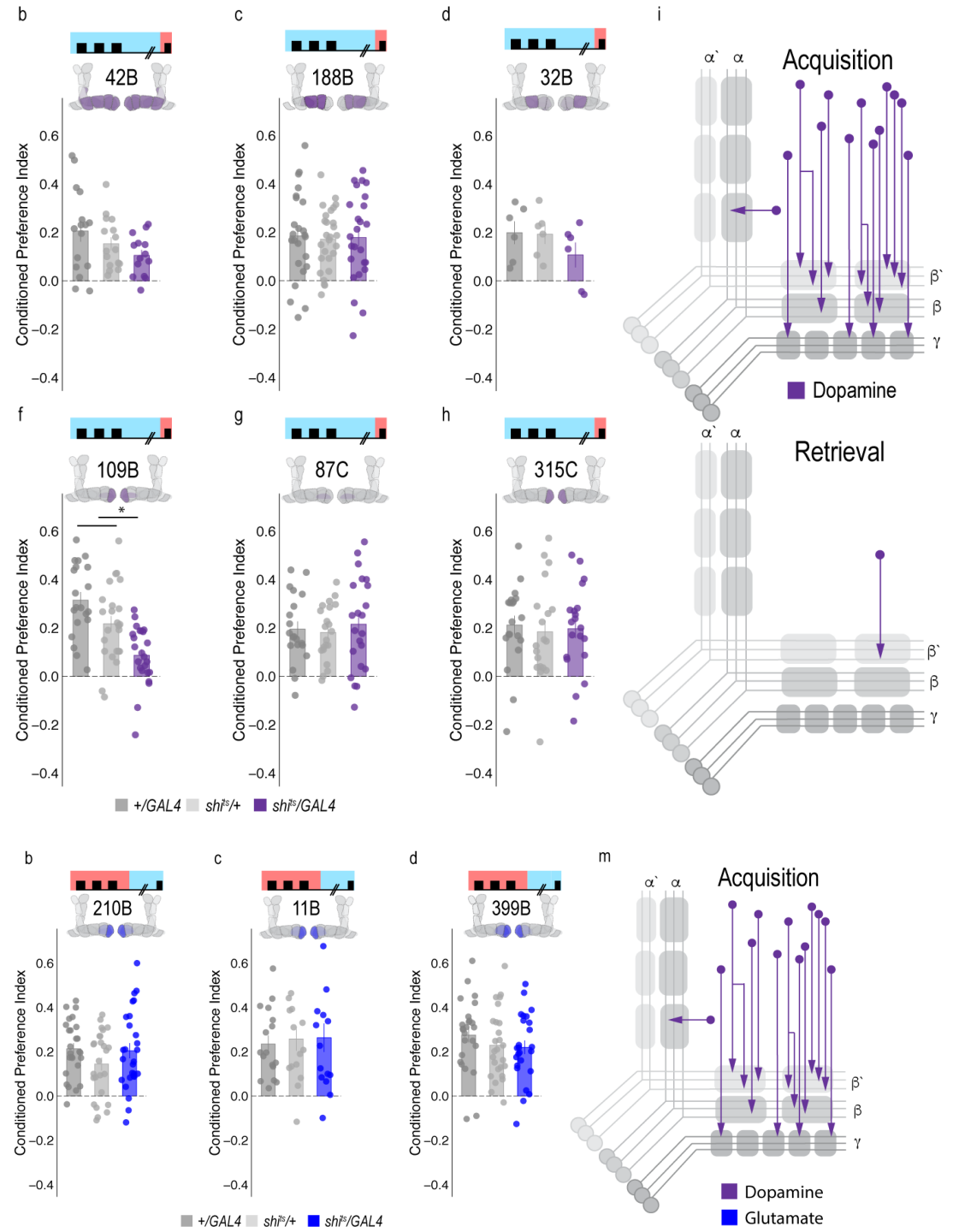

Fig 3. Memory expression during retrieval is dependent on a sparse population of dopamine neurons. (ah) Thermogenetic were used to inactivate neurotransmission during retrieval, but not acquisition, in PAM dopaminergic neurons with varying expression patterns. (f) Inactivating $\beta^{\prime} 2 a$ dopamine neurons during retrieval significantly reduced preference for alcohol associated cues. Bar graphs illustrate mean +/- standard error of the mean. Raw data are overlaid on bar graphs. Each dot is an $n$ of 1 , which equals approximately 60 flies (30 per odor pairing). One-way ANOVA with Tukey Posthoc was used to compare mean and variance. ${ }^{*} p<0.01$ (i) Model of circuits responsible for encoding alcohol associated preference during acquisition and circuits responsible for the expression of alcohol associated preference during retrieval. This model highlights the importance of population level dopaminergic activity during acquisition, whereas sparse subsets of dopaminergic activity are important during retrieval for the expression of alcohol associated preference.

Fig 4. Memory expression during retrieval, but not acquisition, is dependent on a sparse population of glutamatergic MBONs. (a-d) Thermogenetic inactivation of glutamatergic MBONs innervating similar compartments to $\beta^{`} 2 a$ PAM dopaminergic neurons during acquisition did not disrupt encoding of alcohol associated preference. (e-h) However, inactivating neurotransmission during retrieval revealed the specific importance of MBON $\beta 2 \beta$ ' 2 a glutamatergic neuron for the expression of alcohol associated preference. (i-I) Knockdown of dopamine receptors in MBON $\beta 2 \beta^{\prime} 2 a$ did not disrupt alcohol associated preference suggesting that dopamine and $\mathrm{MBON}$ glutamate neurons interact indirectly via the MB. Bar graphs illustrate mean +/- standard error of the mean. Raw data are overlaid on bar graphs. Each dot is an $n$ of 1 , which equals approximately 60 flies (30 per odor pairing). One-way ANOVA with Tukey Posthoc was used to compare mean and variance. ${ }^{*} p<0.01$ (m) Updated model of circuits responsible for encoding alcohol associated preference during acquisition and circuits responsible for the expression of alcohol associated preference during retrieval. Retrieval circuits require specific subsets of dopaminergic neurons and a single MBON glutamatergic neuron innervating the $\beta 2$ 'a compartment. 
bioRxiv preprint doi: https://doi.org/10.1101/578401; this version posted March 15,2019 . The copyright holder for this preprint (which was not certified by peer review) is the author/funder, who has granted bioRxiv a license to display the preprint in perpetuity. It is made available under aCC-BY-ND 4.0 International license.

Scaplen et al., 15 Mar 2019 - preprint copy - BioRxiv

neurons, the paired posterior lateral 1 (PPL1) population, which is responsible for assigning negative valences to associated cues (Aso, Herb et al. 2012, Waddell 2013). This suggests that a second microcircuit in the vertical lobe, which converges onto the $\beta$ ' $2 \mathrm{mp}$ neuron, is important for alcohol associated preference.

Interestingly, trans-Tango did not identify the $\alpha 2$ cholinergic MBON as a postsynaptic target of $\alpha 2 \alpha^{\prime} 2$ dopaminergic neuron. Similarly, RNAi against D1-like receptors or D2-like receptors did not disrupt alcohol associated preference (Figure 6f-h), suggesting that either these subsets of neurons are not directly connected or their direct connectivity is not required for alcohol associated preference. This type of connectivity is striking because it suggests that microcircuits important for the expression of memory converge on a neuron whose activity regulates consolidation, which might underlie memory flexibility. Future studies should address how the relative activity of $\beta$ ' $2 \mathrm{mp}$ can augment memory expression.
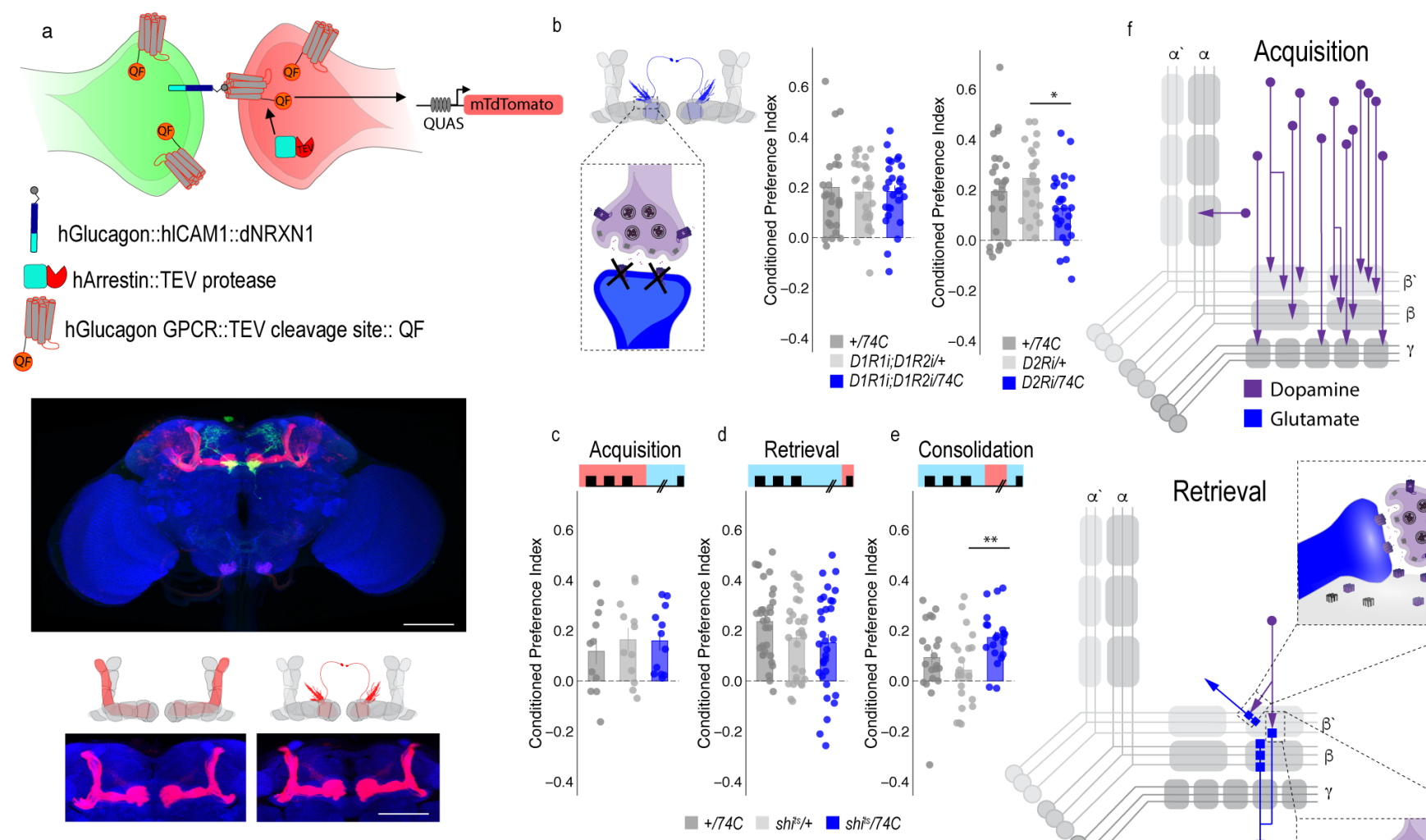

\section{Alcohol memory expression circuits converge on a higher-order integration center}

Thus far we have identified two MB microcircuits, one in the horizontal lobe that consists of PAM dopaminergic neurons and glutamatergic MBONs and one in the vertical lobe that consists of PPL1 dopaminergic neurons and cholinergic MBONs. Interestingly these two microcircuits converge on to a separate glutamatergic $\mathrm{MBON}\left(\beta^{\prime} 2 \mathrm{mp}\right)$ that is important for arousal and when inactivated, facilitates consolidation (Figure 6i). This suggests a circuit framework through which alcohol could shift memory from flexible to inflexible. However, it is still unclear how the MB and MBON activity drive goal directed behavior in the fly. Emerging models in the MB field suggest that MBON activity is pooled across compartments and that learning shifts the balance of activity to favor approach or avoidance (Owald and Waddell 2015). It remains unclear where this $\mathrm{MBON}$ activity converges.

In order to identify potential regions that integrated MBON activity, we used trans-Tango to map postsynaptic partners of $\alpha^{\prime} 2, \beta^{`} 2 \mathrm{mp}$, and 
bioRxiv preprint doi: https://doi.org/10.1101/578401; this version posted March 15,2019 . The copyright holder for this preprint (which was not certified by peer review) is the author/funder, who has granted bioRxiv a license to display the preprint in perpetuity. It is made available under aCC-BY-ND 4.0 International license.

Scaplen et al., 15 Mar 2019 - preprint copy - BioRxiv

$\beta 2 \beta$ 2a MBONs. As mentioned previously, $\alpha$ `2 MBON labeled with MB018B-split-GAL4 identified $\beta^{`} 2 \mathrm{mp}$ as a postsynaptic target. In a number of flies, $\gamma^{5} \beta^{`} 2 \mathrm{a}$ was also identified as a target, however, this was less consistent. Interestingly, the dorsal regions of the FSB, specifically layers $4 / 5$ or layer 6 , were consistently identified as postsynaptic target of $\alpha^{\prime} 2$ (Figure $7 \mathrm{a}, \mathrm{c}$ ). Strikingly both $\beta$ ' $2 \mathrm{mp}$ and $\beta 2 \beta$ ' 2 a also have synaptic connectivity with the dorsal regions of the FSB (Figure 7b, d). Together these data reveal the dorsal FSB as an intriguing convergent region downstream of the $\mathrm{MB}$ whose role in alcohol associated preference should be investigated further (Figure 7e).

\section{Discussion}

A classic hallmark of addiction is the enduring propensity to relapse despite prolonged abstinence. Often relapse is driven by drug associated cues that evoke powerful and pervasive memories. Here we address the mechanisms underlying this persistence by investigating how these memories are encoded and expressed using a combination of behavioral, thermogenetic, in vivo calcium imaging and genetic transsynaptic tracing approaches in the fruit fly, Drosophila melanogaster.

Alcohol is a unique stimulus, because unlike natural rewards or punishers, it has both aversive and appetitive properties and yet the long-standing memories of intoxicating experiences are enduringly appetitive. Strikingly, the expression of this preference is not immediate. When flies are presented with a choice between the
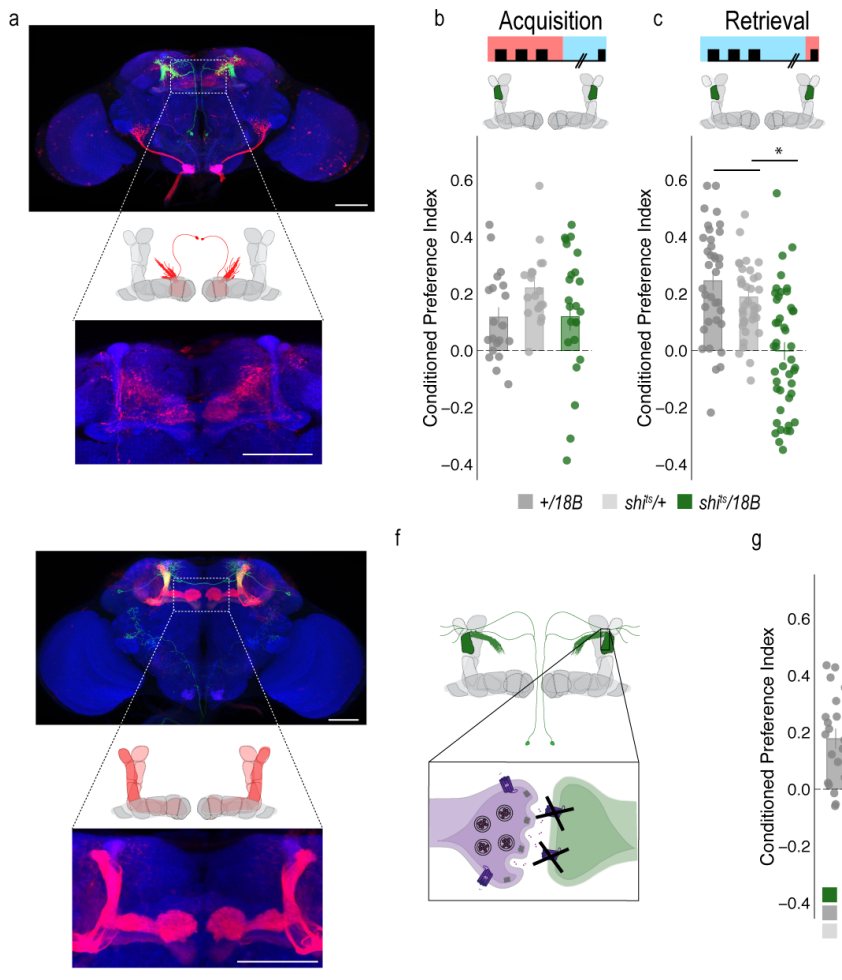

g unpaired odor and the paired odor 30 minutes following acquisition of alcohol memory, flies avoid the paired odor and instead show a preference for cues not associated with alcohol (Kaun, Azanchi et al. 2011). Interestingly, cue associated avoidance switches to an enduring preference 24 hours later. The temporal nature of this valence switch suggests that, like mammals, the neural circuitry underlying the acquisition and expression of cue associated preference in flies is both anatomically complex, temporally distinct, and involves long-lasting changes to the circuitry mechanism.

Here we show that contrary to adaptive aversive or appetitive memories in flies (Liu, Placais et al. 2012, Masek, Worden et al. 2015, Yamagata, Ichinose et al. 2015, Yamagata, Hiroi et al. 2016), encoding alcohol associated preference is not dependent on a single subset of dopamine neurons or output neurons projecting from the MB, but a population of dopamine neurons whose activity emerges over the course of exposure to intoxicated doses of alcohol. We postulate that an increased requirement of dopamine neurons contributes to the stability of alcohol memory. Similarly, increasing the number of DA neurons that encode an aversive stimulus enhances how long memory lasts in Drosophila (Aso and Rubin 2016). This suggests a general rule where stability of memory is encoded by the number of dopamine neurons involved during acquisition. Most drugs of abuse initially increase dopamine levels beyond what is experienced during natural reward (Nutt, Lingford-Hughes et al. 2015, Volkow and Morales 2015, Kegeles, Horga et al. 2018). Our data suggests that this likely occurs, at least in part, via recruitment of additional dopamine neurons. Understanding the mechanism by which dopamine neurons are recruited may provide
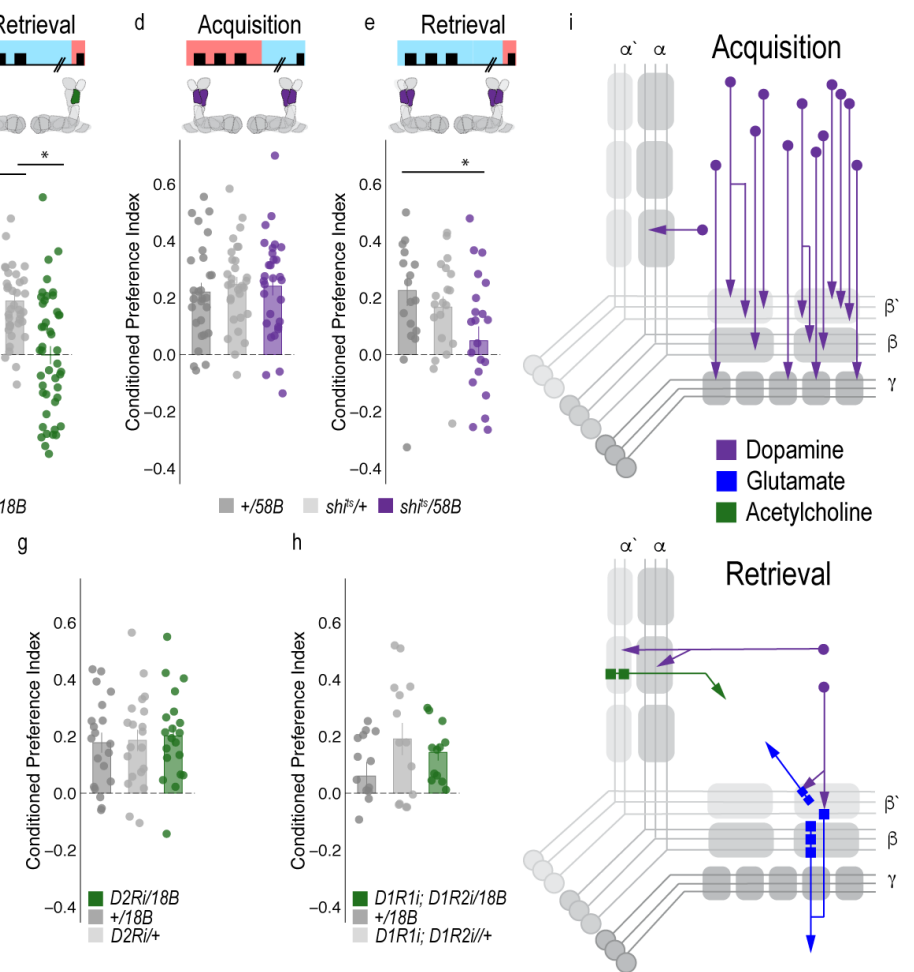

Fig. 6. A microcircuit within the vertical lobe is important for alcohol associated preference. (a) trans-Tango experiments confirms that $\beta$ ' $2 \mathrm{mp}$ glutamatergic MBON is postsynaptic to $\alpha^{\prime} 2$ cholinergic neuron. (b-c) Thermogenetic inactivation of $\alpha^{`} 2$ cholinergic neurons during acquisition did not affect the expression of alcohol associated preference, however, inactivation during retrieval significantly reduced preference. (d-e) Similarly, thermogenetic inactivation of $\alpha 2 \alpha^{\prime} 2$ dopaminergic neurons during acquisition did not affect the expression of alcohol associated preference, however, inactivation during retrieval significantly reduced preference. (f) trans-Tango experiments reveal that $\alpha 2 \alpha^{\prime} 2$ dopaminergic neurons are not synaptically connected to $\alpha^{\prime} 2$ cholinergic MBON. (g-i) Knockdown of either D2R receptors or D1R receptors did not affect alcohol associated preference. (j) Updated model of circuits responsible for encoding alcohol associated preference during acquisition and circuits responsible for the expression of alcohol associated preference during retrieval. Scale bar $=50 \mu \mathrm{m}$ 


\section{a}

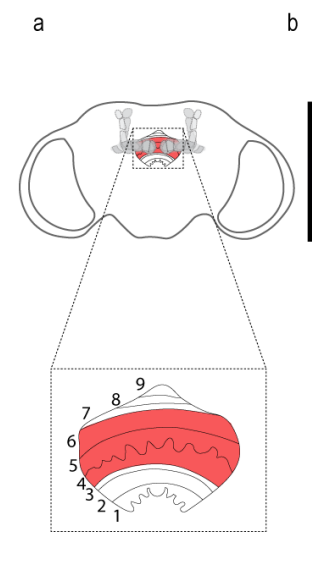

b

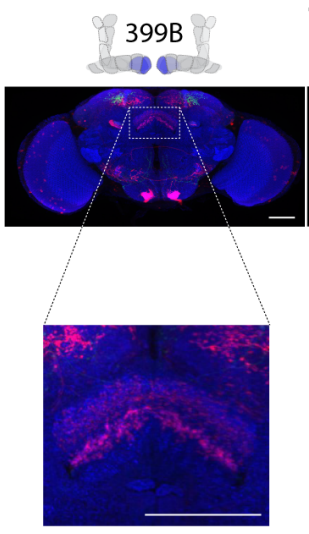

e

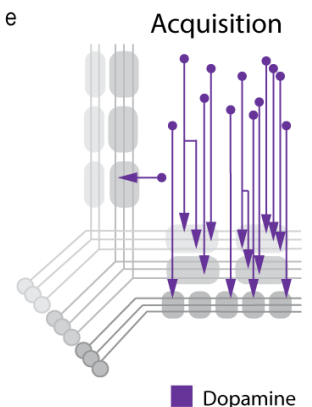

C

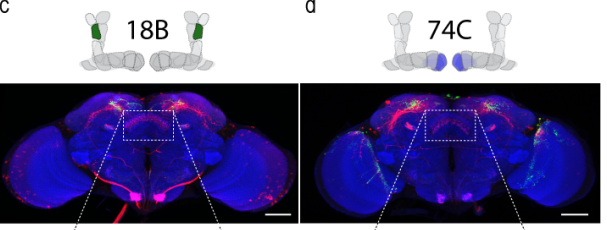

7. Circuits important for memory expression at retrieval converge on the dorsal FSB. (a) Schematic of fly brain with FSB and its layers highlighted. The FSB is a 9-layer structure (Wolff, lyer et al. 2015), of which layers 4,5 , and 6 are targets. (b) Confocal stack of FSB highlighting the postsynaptic signal of $\beta 2 \beta{ }^{\wedge} 2 a$ MBON in the FSB. This MBON predominately targets layers 4 , and 6 . (c) Confocal stack of FSB highlighting the postsynaptic signal of $\alpha^{\prime} 2 \mathrm{MBON}$ in the FSB. This MBON predominately targets layer 6 . (d) Confocal stack of FSB highlighting the postsynaptic signal of $\beta^{\prime} 2 \mathrm{mp}$ MBON in the FSB. This MBON predominately targets layer 4 and 5 . (e) Updated model of circuits responsible for encoding alcohol associated preference during acquisition and circuits responsible for the expression of alcohol associated preference during retrieval. Model highlights the interconnectivity of the vertical and horizontal microcircuits and layers within the dorsal FSB where MBONs converge. Scale bar = $50 \mu \mathrm{m}$

powerful insight into why memories for an intoxicating experience are so persistent.

In order to understand the mechanism of memory persistence, it is necessary to understand how dopamine encodes memories for cues associated with reward. In both primates and rodents, studies report a distinctive response profile whereby dopamine neuronal activity shifts its response from the presentation of a reward during learning to the presentation of a reward-predictive cue during expression (Keiflin and Janak 2015, Schultz 2016). However, given the heterogeneity of neurons in the VTA and the fact that these responses are typically observed using extracellular recordings, it is unclear how many and which dopamine neurons within the VTA and SNc exhibit this distinctive response to reward-predictive cues. Our intersectional genetic approach in Drosophila provided the temporal and spatial resolution to address this question. We found responses to rewardpredictive cues were restricted to a small subset of dopamine neurons that were active during the initial alcohol presentation. These data suggest that cue responses are sparsely represented within a discrete subset of dopamine neurons found within the larger population of reward encoding dopamine neurons. Having identified a subset of alcohol associated cue-responsive dopamine neurons, we are now poised to further examine how the activity pattern of these neurons change with time and experience in vivo.

Additionally, we found cue-responsive dopamine neurons make direct connections with a glutamatergic neuron implicated in arousal (Sitaraman, Aso et al. 2015). Blocking this $\beta$ ' $2 \mathrm{mp}$ neuron when flies normally sleep enhanced memory in a D2R-dependent manner. We propose that $\beta^{\prime} 2 \mathrm{a}$ dopamine neurons inhibit $\beta$ ’ $2 \mathrm{mp}$ glutamate neuronal activity, thus permitting consolidation of alcohol associated preference. Strikingly, $\beta^{`} 2 \mathrm{a}$ dopaminergic neurons were previously reported to inhibit the output of $\beta ` 2$ amp MBONs to promote approach behaviors when flies were presented with conflicting aversive and appetitive odor cues (Lewis, Siju et al. 2015). Like other animals, flies find $\mathrm{CO}_{2}$ aversive, however, in the context of decaying fruit, flies often approach appetitive food odor cues despite the innately aversive $\mathrm{CO}_{2}$ odor cues. Lewis et al (2015) demonstrated $\beta ` 2 a$ dopaminergic neurons activity was required to overcome the innately aversive properties of $\mathrm{CO}_{2}$ and exhibit preference for these combined odor cues. The effects of $\beta$ ' $2 \mathrm{a}$ dopamine neuronal inhibition, however, were not long lasting and thus responses to $\mathrm{CO}_{2}$ remained flexible outside the context of appetitive food odors. Here, the appetitive food odor, and consequently the activity of $\beta$ 2a dopaminergic neurons, appears to act as an occasion setter, or a discriminatory stimulus that augments an animal's response to a cue, thereby changing fly's typical response to $\mathrm{CO}_{2}$ (Lewis, Siju et al. 2015). We speculate this neuron also resets the response to a cue associated with alcohol, which may be critical for overcoming the initial aversive properties of alcohol. We postulate that repeated intoxicating experiences change the dynamics of $\beta^{\prime} 2 \mathrm{a}$ dopamine neurons during acquisition or consolidation in a way that create long term changes to the responsivity of the $\beta 2 \mathrm{mp}$ MBON. This ultimately results in an inflexible memory circuit that supports alcohol associated preference.

Once acquired, distinct processes regulate consolidation and expression of enduring preferences for cues associated with alcohol intoxication. We found this process relies on a complex multilevel neural circuit that extends across brain regions to integrate information and consist of two converging microcircuits, each with its own subset of dopaminergic neurons and MBONs. These microcircuits emerge with time, are not necessary for the acquisition of these long-lasting preference associations, and converge on the $\beta$ ' $2 \mathrm{mp}$ glutamate neuron (Figure 8 ). The $\beta$ ' $2 \mathrm{a}$ dopamine neurons, which innervate the $\beta^{\prime} 2 \mathrm{mp}$ glutamate neuron, make indirect connections through odor-coding MB Kenyon 
bioRxiv preprint doi: https://doi.org/10.1101/578401; this version posted March 15, 2019. The copyright holder for this preprint (which was not certified by peer review) is the author/funder, who has granted bioRxiv a license to display the preprint in perpetuity. It is made available under aCC-BY-ND 4.0 International license.

Scaplen et al., 15 Mar 2019 - preprint copy - BioRxiv

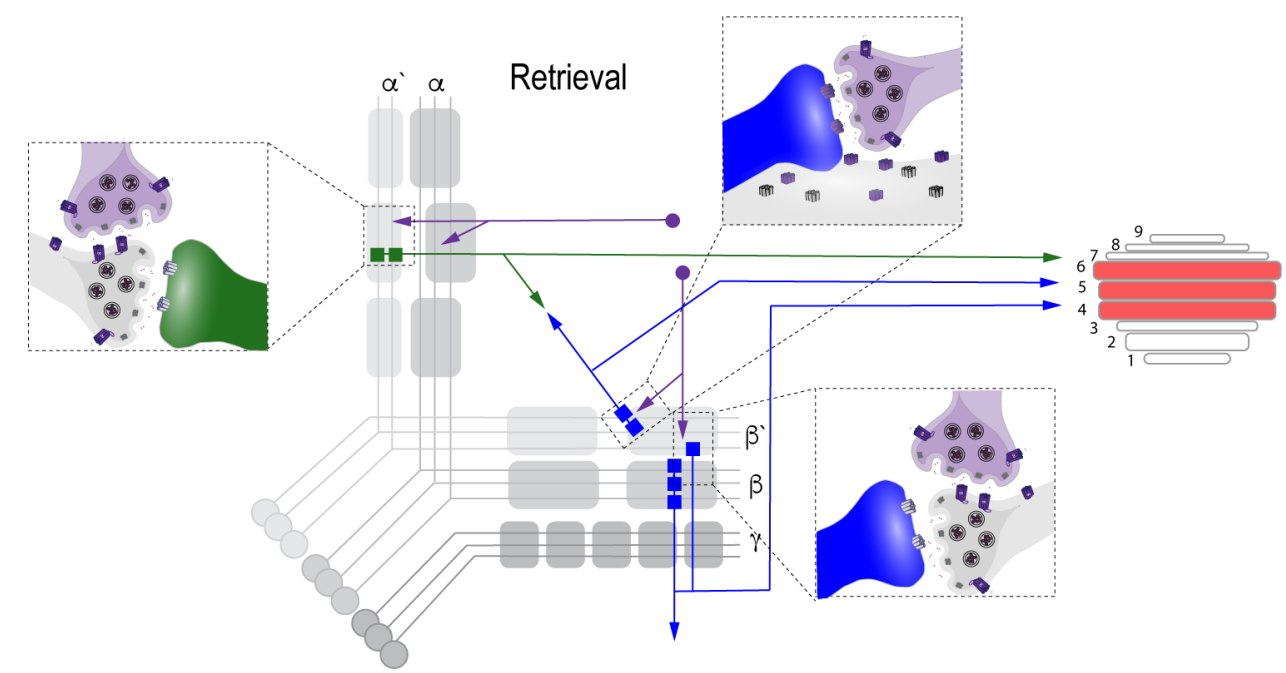

Fig 8. Proposed circuit important for the expression of alcohol associated preference at retrieval. The proposed circuit comprises two separate microcircuits, one in the vertical lobe and one in the horizontal lobe that converge on $\beta^{2} 2 \mathrm{mp}$ MBON as well as layers with the dorsal FSB. The vertical lobe microcircuit includes a PPL1 $\alpha^{\prime} 2 \alpha 2$ dopaminergic neuron that have indirect connections with an $\alpha^{\prime} 2$ cholinergic MBON via the MB. The horizontal lobe microcircuit includes a subset of PAM dopaminergic neurons $\left(\beta^{\prime} 2 a\right)$ that have direct connections with the $\beta^{\prime} 2 \mathrm{mp}$ glutamatergic MBON and indirect connections with the $\beta 2 \beta^{\prime} 2 a$ glutamatergic MBON. Vertical and horizontal microcircuits converge on the $\beta^{\wedge} 2 \mathrm{mp}$ glutamatergic MBON which is important for arousal (Sitaraman, Aso et al. 2015) and layers 4,5 , and 6 of the FSB.

cells to the $\beta 2 \beta$ ' 2 a glutamate neurons. Similarly, the $\alpha 2 \alpha$ ' 2 dopamine neurons make indirect connections with the $\alpha^{\prime} 2$ cholinergic neuron, which also innervates the $\beta$ ' $2 \mathrm{mp}$ neuron. The requirement for dopamine neurons implicated in encoding both reward and punishment in the expression of alcohol memory speaks to how seemingly conflicting events are integrated to produce an appropriate behavioral response.

Our data suggest that activity of these $\beta$ '2a and $\alpha^{\prime} 2$ dopamine-MBON microcircuits is required for expression of alcohol associated preference. Unlike sucrose or shock memory formation where dopamine neurons inhibit the response of MBONs to elicit an appropriate behavioral response following training (Perisse, Yin et al. 2013, Aso, Sitaraman et al. 2014, Hige, Aso et al. 2015, Owald, Felsenberg et al. 2015, Aso and Rubin 2016, Cognigni, Felsenberg et al. 2018 ), our data suggest that activation of both the dopamine and output neurons in $\beta$ ' $2 \mathrm{a}$ and $\beta$ ' 2 compartment are required to initiate an alcohol seeking behavioral response. Because the $\beta^{\prime} 2 \mathrm{mp}$ neuron is not required for expression of memory, it is likely that its output is integrated elsewhere in the brain to drive goal directed behaviors.

Further investigation of downstream targets of these microcircuits identified an additional convergent region: the dorsal layers of the FSB, specifically layers 4, 5, and 6. The identification of the FSB as a convergence region of MBONs is notable because a popular model proposes that learned behavioral responses in the fly are a consequence of pooled activity of mushroom body output neurons, which shifts the gain to either approach or avoidance behavior (Owald and Waddell 2015). Here we have identified one such structure that is an anatomical candidate for pooling MB output activity to drive learned behaviors. Interestingly, although the FSB has an established role in arousal and sleep, more recent work has defined its role in innate and learned nociceptive avoidance further supporting its role in integrating $\mathrm{MB}$ output activity (Hu, Peng et al. 2018). We hypothesize that output signals from the $\beta 2 \beta^{\prime} 2 \mathrm{a}$ and $\alpha^{\prime} 2$ neurons are integrated at the FSB to shift naïve response to cue-directed learned response. Compellingly, the $\beta$ ' $2 \mathrm{mp}$ neuron also sends projections the FSB, presenting a circuit framework through which flexibility of memory could influence memory expression.

Together, these results provide valuable insight to the dynamic qualities of memory and how the long-lasting reward memories for alcohol intoxication are acquired, maintained, and expressed. We demonstrate that appetitive response for alcohol is encoded within a population of dopamine neurons, and expressed through microcircuits defined by subsets of these dopamine neurons. These microcircuits converge at several points; one through a neuron that regulates consolidation, and another at a downstream target implicated in integration of naïve and learned responses. This provides a new framework for how drugs of abuse regulate acquisition and expression of sensory memories, which ultimately results in a shift in behavioral response from malleable to inflexible.

\section{Materials and Methods}

\section{Fly Strains}

All Drosophila melanogaster lines were raised on standard cornmeal-agar media with tegosept anti-fungal agent and maintained at either $18^{\circ} \mathrm{C}$ or $21^{\circ} \mathrm{C}$. For a list of fly lines used in the study, see Table 1. All Drosophila melanogaster lines used for trans-Tango were raised and maintained at $18^{\circ} \mathrm{C}$ in humidity-controlled chambers under $14 / 10 \mathrm{hr}$ light/dark cycles on standard cornmeal-agar media with tegosept anti-fungal agent.

\section{Behavioral Experiments}

Odor Preference Conditioning: For behavior experiments, male flies were collected 1-2 days post eclosion and were shifted from $21^{\circ} \mathrm{C}$ to $18^{\circ} \mathrm{C}$, $65 \%$ humidity and placed on a $14 / 10 \mathrm{hr}$ light/dark cycle. Odor conditioning was performed similar to Kaun et al. 2011. In short, groups of 30 males were trained in perforated $14 \mathrm{ml}$ culture vials filled with $1 \mathrm{ml}$ of $1 \%$ agar and covered with mesh lids. Training rooms were temperature and humidity controlled $(65 \%)$. Training was performed in the dark with minimal red-light illumination and was preceded by a 20- minute habituation to the training chambers. Training chambers were constructed out of PlexiGlas $(30 \times 15 \times 15 \mathrm{~cm}$ ) (for details please refer to (Nuñez, Azanchi et al. 2018)). During habitation, humidified air (flow rate: 130) was streamed into the chambers. A single training session consisted of a 10 minute presentation of odor 1 (flow rate: 130), followed by a 10 minute presentation of odor 2 (flow rate 130 ) with $60 \%$ ethanol (flow rate 90: ethanol 60:air). Reciprocal training was performed simultaneously to ensure that inherent preference for either odor did not affect conditioning scores. For the majority of experiments odors used were 1:36 isoamyl alcohol and 1:36 isoamyl acetate in mineral oil, however, screen behavioral experiments used 1:36 isoamyl alcohol and 1:36 ethyl acetate in mineral oil. Vials of flies from Group 1 and Group 2 were age matched and paired according to placement in the training chamber. Pairs were tested simultaneously 24 hours later in the $Y$ maze by streaming odor 1 and odor 2 (flow rate 10) in separate arms and allowing flies to walk up vials to choose between the two arms. A preference index was calculated by \# flies in the Paired Odor Vial- \# flies in the Unpaired Odor Vial)/ total \# of flies that climbed. A conditioned preference index (CPI) was calculated by the averaging preference indexes from reciprocal groups. All data are reported as CPI. All plots were generated in RStudio. 
bioRxiv preprint doi: https://doi.org/10.1101/578401; this version posted March 15, 2019. The copyright holder for this preprint (which was not certified by peer review) is the author/funder, who has granted bioRxiv a license to display the preprint in perpetuity. It is made available under aCC-BY-ND 4.0 International license.

Scaplen et al., 15 Mar 2019 - preprint copy - BioRxiv

Odor Sensitivity: Odor sensitivity was evaluated at restrictive temperatures $\left(30^{\circ} \mathrm{C}\right)$. Odors used were 1:36 iso-amyl alcohol in mineral oil and 1:36 is-amyl acetate in mineral oil. Groups of 30 naïve males were presented with either an odor (flow rate 10) or air streamed through mineral oil in opposite arms of the Y. Preference index was calculated by \# flies in odor vial- \# flies in air vial)/ total \# flies that climbed for each individual odor.

Ethanol Sensitivity: Ethanol sensitivity was evaluated in the recently developed flyGrAM assay (Scaplen and Mei et al 2019). Briefly, for thermogenetic inactivation, 10 flies were placed into arena chambers and placed in a $30^{\circ} \mathrm{C}$ incubator for 20 minutes prior to testing. The arena was then transferred to a preheated $\left(30^{\circ} \mathrm{C}\right)$ light sealed box and connected to a vaporized ethanol/humidified air delivery system. Flies were given an additional 15 minutes to acclimate to the box before recordings began. Group activity was recorded ( 33 frames $/ \mathrm{sec}$ ) for five minutes of baseline, followed by 10 minutes of ethanol administration and five minutes of following ethanol exposure. Activity was binned by 10 seconds and averaged within each genotype. Mean group activity is plotted as a line across time with standard error of the mean overlaid. All activity plots were generated in RStudio.

\section{trans-Tango Immunohistochemistry and Microscopy}

Experiments were performed according to the published FlyLight protocols with minor modifications. Briefly, either adult flies that are 15-20 days old were cold anaesthetized on ice, de-waxed in $70 \%$ ethanol dissected in cold Schneider's Insect Medium (S2). Within 20 minutes of dissection, tissue was incubated in 2\% paraformaldehyde (PFA) in S2 at room temperature for 55 minutes. After fixation, samples were rinsed with phosphate buffered saline with $0.5 \%$ Triton X-100 (PBT) and washed 4 times for 15 minutes at room temperature. Following PBT washes, PBT was removed and samples were incubated in SNAP substrate diluted in PBT (SNAP-Surface649, NEB S9159S; 1:1000) for 1 hour at room temperature. Samples were then rinsed and washed 3 times for 10 minutes at room temperature and then blocked in $5 \%$ heat-inactivated goat serum in PBT for 90 minutes at room temperature and incubated with primary antibodies (Rabbit a-GFP Polyclonal (1:1000), Life Tech \#A11122, Rat $\alpha-H A$ Monoclonal (1:100), Roche \#11867423001) for two overnights at $4^{\circ} \mathrm{C}$. Subsequently, samples were rinsed and washed 4 times for 15 minutes in $0.5 \%$ PBT and incubated in secondary antibodies (Goat a-Rabbit AF488 (1:400), Life Tech \#A11034, Goat a-Rat AF568 (1:400), Life Tech \#A11077) diluted in 5\% goat serum in PBT for 2-3 overnights at $4^{\circ} \mathrm{C}$. Samples were then rinsed and washed 4 times for 15 minutes in $0.5 \%$ PBT at room temperature and prepared for DPX mounting. Briefly, samples were fixed a second time in 4\% PFA in PBS for 4 hours at room temperature and then washed 4 times in PBT at room temperature. Samples were rinsed for 10 minutes in PBS, placed on PLLdipped cover glass, and dehydrated in successive baths of ethanol for 10 minutes each. Samples were then soaked 3 times in xylene for 5 minutes each and mounted using DPX. Confocal images were obtained using a Zeiss, LSM800 with ZEN software (Zeiss, version 2.1) with auto Z brightness correction to generate a homogeneous signal where it seemed necessary, and were formatted using Fiji software (http://fiji.sc).

\section{Dopamine Immunohistochemistry and Microscopy}

Groups of flies were exposed to either 10 minutes of air or 10 minutes of ethanol and dissected within 15 minutes of exposure on ice. Immunohistochemistry was performed according to (Cichewicz, Garren et al. 2017). With 15 minutes of dissection, tissue was transferred to fix $(1.25 \%$ glutaraldehyde in $1 \% \mathrm{PM})$ for $3-4$ hours at $4^{\circ} \mathrm{C}$. Tissue was subsequently washed 3 times for 20 minutes in PM and reduced in $1 \%$ sodium borohydride. Then the tissue was washed 2 times for 20 minutes before a final wash in PMBT. Tissue was blocked in $1 \%$ goat serum in PMBT overnight at $4{ }^{\circ} \mathrm{C}$ and incubated in primary antibody (Mouse antidopamine (1:40) Millipore Inc, \#MAB5300) for 48 hours at $4^{\circ} \mathrm{C}$. Following primary antibody incubation, tissue was washed 3 times in PBT for 20 minutes at room temperature and incubated in secondary antibody (Goat anti mouse $488\left(1: 200\right.$ in PBT) Thermo \#A11029) for 24 hours at $4^{\circ} \mathrm{C}$. The following day tissue was washed 2 times for 20 minutes in PBT and then overnight in fresh PBT. Tissue was rinsed quickly in PBS, cleared in FocusClear and mounted in MountClear (Cell Explorer Labs). Confocal images were obtained using a Zeiss, LSM800 with ZEN software (Zeiss, version 2.1). Microscope settings were established using ethanol tissue before imaging air and ethanol samples.

\section{Dopamine fluorescence analysis}

Fluorescence was quantified in Fiji (Schindelin, Arganda-Carreras et al. 2012) using Segmentation Editor and 3D Manager (Ollion, Cochennec et al. 2013). In segmentation editor ROls were defined using the selection tool brush to outline the MB in each slice and also outside a background region immediately ventral to $M B$ that lacked defined fluorescent processes. 3D ROls of the $\mathrm{MB}$ and control region were created by interpolating across slices. Geometric and intensity measurements were calculated for each ROI in 3D Manager and exported to CSV files. Integrated density for each ROI was normalized by the integrated density of control regions. Average integrated density for air and ethano exposures are reported. All fluorescence quantifications were performed by a blinded experimenter.

\section{Calcium Imaging Protocol and Analysis}

To express GCaMP6m in PAM neurons, UAS-GCaMP6m virgin female flies were crossed to male flies containing the R58E02-GAL4 driver. As previously mentioned, all flies were raised on standard cornmeal agar food media with tegosept anti-fungal agent and maintained on a 14/10hour light/dark cycle at $24^{\circ} \mathrm{C}$ and $65 \%$ humidity.

Fly Preparation. Male flies were selected for imaging six days posteclosion. Flies were briefly anesthetized on ice to transfer and fix to an experimental holder made out of heavy-duty aluminum foil. The fly was placed into an $\mathrm{H}$-shaped hold cut out of the foil and glued in place using epoxy (5-min Epoxy, Devcon). The head was tilted about $70^{\circ}$ to remove the cuticle from the back of the fly head. All legs were free to move, the proboscis and antenna remained intact and unglued. Once the epoxy was dry, the holder was filled with Drosophila Adult Hemolymph-Like Saline (AHLS). The cuticle was removed using a tungsten wire (Roboz Surgical Instruments Tungsten Dissecting Needle, .125 mm, Ultra Fine $(P k$ 10)) and forceps \#5. The prepared fly in its holder was positioned on a customized stand underneath the two-photon scope. The position of the ball and the stream delivery tubes were manually adjusted to the fly's position in the holder.

Imaging paradigm. Calcium imaging recordings were performed with a two-photon resonance microscope (Scientifica). Fluorescence was recorded from the PAM neurons innervating the mushroom body for a total duration of 80 to 95 minutes. The first 10 minutes the fly was presented an air stream, followed by 10 minutes of isoamyl alcohol. The fly was then presented with 10-minutes of isoamyl alcohol paired with ethanol followed by 50 minutes of streaming air. To avoid bleaching effects and to match the higher resolution imaging properties, the recording was not throughout the entire paradigm but spaced with imaging intervals of 61.4 seconds. Recordings were performed using SciScan Resonance Software (Scientifica). The laser was operated at $930 \mathrm{~nm}$ wavelength at an intensity of $7.5-8 \mathrm{~mW}$. Images were acquired at $512 \times 512$-pixel resolution with an average of 30.9 frames per second Recordings lasted 1900 frames which equals 61.5 seconds. Recordings were performed at $18.5^{\circ} \mathrm{C}$ room temperature and $59 \%$ humidity.

Imaging analysis. Data were registered, processed, and extracted using a custom matlab GUI (Chris Deister github.com/cdesiter). Calcium image files (.tiff) comprising of 1900 frames taken at 30.94 frames per second rate (61.4 seconds), were initially averaged every 5 frames to downsize the tiff image files to 380 frames. Image files were then aligned and registered in X-Y using a 15-50 frame average as a template. ROls were constructed over the MB lobes using non-negative matrix factorization to identify active regions and then subsequently segmented to create the ROls. Fluorescence values were extracted from identified ROls and $\Delta \mathrm{F} / \mathrm{F}_{\mathrm{o}}$ measurements were created using a moving-average of 75 frames to calculate the baseline fluorescence $\left(F_{0}\right)$. Average fluorescence traces across flies $(\mathrm{n}=6)$ were visualized using ggplot in $\mathrm{R}$ studio. Fiji (Schindelin, Arganda-Carreras et al. 2012) was used to construct heatmaps visualizing calcium activity. Calcium image files were summated across 1900 frames to create Z-projections. A heat gradient was used to visualize calcium activity magnitude.

\section{$q R T-P C R$}

qRT-PCR methods have been described previously (Petruccelli, Feyder et al. 2018). In brief, total RNA was extracted from approximately 100 heads using Trizol (Ambion, Life Technologies) and treated with DNase (Ambion DNA-Free Kit). Equal amounts of RNA (1 $\mu \mathrm{g})$ were reversetranscribed into CDNA (Applied Biosystems) for each of the samples. Then, Biological (R3) and technical (R2) replicates were analyzed with Sybr Green Real-Time PCR (BioRad, ABI PRISM 7700 Sequence Detection System) performed using the following PCR conditions: $15 \mathrm{~s}$ $95^{\circ} \mathrm{C}, 1 \mathrm{~min} 55^{\circ} \mathrm{C}, 40 \mathrm{x}$. Primer sequences can be found in Supplementary Table 4. Across all samples and targets, $\mathrm{Ct}$ threshold and amplification start/stop was set to 0.6 and manually adjusted respectively. All target genes were initially normalized to CG13646 expression for comparative $\Delta \mathrm{Ct}$ method analysis, then compared to control genotype to assess fold enrichment ( $\Delta \Delta \mathrm{Ct}$ method) 
bioRxiv preprint doi: https://doi.org/10.1101/578401; this version posted March 15,2019 . The copyright holder for this preprint (which was not certified by peer review) is the author/funder, who has granted bioRxiv a license to display the preprint in perpetuity. It is made available under aCC-BY-ND 4.0 International license.

Scaplen et al., 15 Mar 2019 - preprint copy - BioRxiv

\section{Declaration of Interests}

The authors declare no conflict of interest.

This article contains supporting information online.

\section{Acknowledgments}

This work was supported by the Smith Family Award Program for Excellence in Biomedical Research, United States, the Israel Binational Science Foundation Start Up Grant 2015005, the Carney Institute for Brain Science Center for Biomedical Research Excellence "Center for Nervous System Function" (NIGMS P20GM103645), NIAAA (R01AA024434), and the Rhode Island Foundation Medical Research Fund 20144133. We thank all of the Kaun lab members for fruitful discussions and feedback on earlier versions of this manuscript. We also thank the Drosophila community, particularly Yoshinori Aso and Gerry Rubin, the Bloomington Stock Center, and the Vienna Drosophila RNAi Center for sharing fly stocks. Yoshinori Aso also provided confocal stacks of MB042B and MB040B used for cell counting. We thank Thomas Boudier and Stefan Helfrich for helpful software advice, Jay Hirsh for reagents and advice, and Christopher Diester and other members of the Moore lab for calcium analysis guidance. Finally, we thank John McGeary, Tara White, and Daniel Dombeck for helpful comments on earlier version of this manuscript. BioRxiv Template from the Finkelstein Lab was used for formatting this manuscript (https://github.com/finkelsteinlab/BioRxiv-Template).

\section{References}

Aso, Y., D. Hattori, Y. Yu, R. M. Johnston, N. A. lyer, T. T. Ngo, H. Dionne, L. F. Abbott, R. Axel, H. Tanimoto and G. M. Rubin (2014). "The neuronal architecture of the mushroom body provides a logic for associative learning." Elife 3: e04577.

Aso, Y., A. Herb, M. Ogueta, I. Siwanowicz, T. Templier, A. B. Friedrich, K. Ito, H. Scholz and H. Tanimoto (2012). "Three dopamine pathways induce aversive odor memories with different stability." PLoS Genet 8(7): e1002768.

Aso, Y. and G. M. Rubin (2016). "Dopaminergic neurons write and update memories with cell-type-specific rules." Elife 5.

Aso, Y., D. Sitaraman, T. Ichinose, K. R. Kaun, K. Vogt, G. BelliartGuerin, P. Y. Placais, A. A. Robie, N. Yamagata, C. Schnaitmann, W. J. Rowell, R. M. Johnston, T. T. Ngo, N. Chen, W. Korff, M. N. Nitabach, U. Heberlein, T. Preat, K. M. Branson, H. Tanimoto and G. M. Rubin (2014). "Mushroom body output neurons encode valence and guide memory-based action selection in Drosophila." Elife 3: e04580.

Barnstedt, O., D. Owald, J. Felsenberg, R. Brain, J. P. Moszynski, C. B. Talbot, P. N. Perrat and S. Waddell (2016). "Memory-Relevant Mushroom Body Output Synapses Are Cholinergic." Neuron 89(6): 1237-1247.

Bassareo, V., M. A. De Luca, M. Aresu, A. Aste, T. Ariu and G. Di Chiara (2003). "Differential adaptive properties of accumbens shell dopamine responses to ethanol as a drug and as a motivational stimulus." Eur J Neurosci 17(7): 1465-1472.

Brodie, M. S. and S. B. Appel (1998). "The effects of ethanol on dopaminergic neurons of the ventral tegmental area studied with intracellular recording in brain slices." Alcohol Clin Exp Res 22(1): 236-244.

Buschman, T. J. and E. K. Miller (2014). "Goal-direction and top-down control." Philos Trans R Soc Lond B Biol Sci 369(1655).

Cembrowski, M. S., M. G. Phillips, S. F. DiLisio, B. C. Shields, J. Winnubst, J. Chandrashekar, E. Bas and N. Spruston (2018). "Dissociable Structural and Functional Hippocampal Outputs via Distinct Subiculum Cell Classes." Cell 173(5): 1280-1292 e1218.
Chen, T. W., T. J. Wardill, Y. Sun, S. R. Pulver, S. L. Renninger, A. Baohan, E. R. Schreiter, R. A. Kerr, M. B. Orger, V. Jayaraman, L. L. Looger, K. Svoboda and D. S. Kim (2013). "Ultrasensitive fluorescent proteins for imaging neuronal activity." Nature 499(7458): 295-300.

Cichewicz, K., E. J. Garren, C. Adiele, Y. Aso, Z. Wang, M. Wu, S. Birman, G. M. Rubin and J. Hirsh (2017). "A new brain dopaminedeficient Drosophila and its pharmacological and genetic rescue." Genes Brain Behav 16(3): 394-403.

Claridge-Chang, A., R. D. Roorda, E. Vrontou, L. Sjulson, H. Li, J. Hirsh and G. Miesenbock (2009). "Writing memories with lightaddressable reinforcement circuitry." Cell 139(2): 405-415.

Cognigni, P., J. Felsenberg and S. Waddell (2018). "Do the right thing: neural network mechanisms of memory formation, expression and update in Drosophila." Curr Opin Neurobiol 49: 51-58.

da Silva, J. A., F. Tecuapetla, V. Paixao and R. M. Costa (2018). "Dopamine neuron activity before action initiation gates and invigorates future movements." Nature 554(7691): 244-248.

Di Chiara, G. and A. Imperato (1985). "Ethanol preferentially stimulates dopamine release in the nucleus accumbens of freely moving rats." Eur J Pharmacol 115(1): 131-132.

Di Chiara, G., G. Tanda and E. Carboni (1996). "Estimation of in-vivo neurotransmitter release by brain microdialysis: the issue of validity." Behav Pharmacol 7(7): 640-657.

Dietzl, G., D. Chen, F. Schnorrer, K. C. Su, Y. Barinova, M. Fellner, B. Gasser, K. Kinsey, S. Oppel, S. Scheiblauer, A. Couto, V. Marra, K. Keleman and B. J. Dickson (2007). "A genome-wide transgenic RNAi library for conditional gene inactivation in Drosophila." Nature 448(7150): 151-156.

Ding, Z. M., S. M. Oster, S. R. Hall, E. A. Engleman, S. R. Hauser, W. J. McBride and Z. A. Rodd (2011). "The stimulating effects of ethanol on ventral tegmental area dopamine neurons projecting to the ventral pallidum and medial prefrontal cortex in female Wistar rats: regional difference and involvement of serotonin-3 receptors." Psychopharmacology (Berl) 216(2): 245-255.

Dodson, P. D., J. K. Dreyer, K. A. Jennings, E. C. Syed, R. WadeMartins, S. J. Cragg, J. P. Bolam and P. J. Magill (2016). "Representation of spontaneous movement by dopaminergic neurons is cell-type selective and disrupted in parkinsonism." Proc Natl Acad Sci U S A 113(15): E2180-2188.

Donlea, J. M., D. Pimentel, C. B. Talbot, A. Kempf, J. J. Omoto, V. Hartenstein and G. Miesenbock (2018). "Recurrent Circuitry for Balancing Sleep Need and Sleep." Neuron 97(2): 378-389 e374.

Fadardi, J. S., W. M. Cox and A. Rahmani (2016). "Neuroscience of attentional processes for addiction medicine: from brain mechanisms to practical considerations." Prog Brain Res 223: 7789.

Fernandez, M. P. and E. A. Kravitz (2013). "Aggression and courtship in Drosophila: pheromonal communication and sex recognition." $\mathrm{J}$ Comp Physiol A Neuroethol Sens Neural Behav Physiol 199(11): 1065-1076.

Field, M. and W. M. Cox (2008). "Attentional bias in addictive behaviors: a review of its development, causes, and consequences." Drug Alcohol Depend 97(1-2): 1-20.

Font, L. and C. L. Cunningham (2012). "Post-retrieval propranolol treatment does not modulate reconsolidation or extinction of ethanol-induced conditioned place preference." Pharmacol Biochem Behav 101(2): 222-230.

Harris, D. T., B. R. Kallman, B. C. Mullaney and K. Scott (2015). "Representations of Taste Modality in the Drosophila Brain." Neuron 86(6): 1449-1460.

Heisenberg, M. (2015). "Outcome learning, outcome expectations, and intentionality in Drosophila." Learn Mem 22(6): 294-298. 
bioRxiv preprint doi: https://doi.org/10.1101/578401; this version posted March 15,2019 . The copyright holder for this preprint (which was not certified by peer review) is the author/funder, who has granted bioRxiv a license to display the preprint in perpetuity. It is made available under aCC-BY-ND 4.0 International license.

Scaplen et al., 15 Mar 2019 - preprint copy - BioRxiv

Herrero, P. (2012). "Fruit fly behavior in response to chemosensory signals." Peptides 38(2): 228-237.

Hige, T., Y. Aso, M. N. Modi, G. M. Rubin and G. C. Turner (2015). "Heterosynaptic Plasticity Underlies Aversive Olfactory Learning in Drosophila." Neuron 88(5): 985-998.

Hoke, K. L., E. A. Hebets and D. Shizuka (2017). "Neural Circuitry for Target Selection and Action Selection in Animal Behavior." Integr Comp Biol 57(4): 808-819.

Howard, E. C., C. J. Schier, J. S. Wetzel, C. L. Duvauchelle and R. A. Gonzales (2008). "The shell of the nucleus accumbens has a higher dopamine response compared with the core after non-contingent intravenous ethanol administration." Neuroscience 154(3): 10421053.

Howe, M. W. and D. A. Dombeck (2016). "Rapid signalling in distinct dopaminergic axons during locomotion and reward." Nature 535(7613): 505-510.

Hu, W., Y. Peng, J. Sun, F. Zhang, X. Zhang, L. Wang, Q. Li and Y. Zhong (2018). "Fan-Shaped Body Neurons in the Drosophila Brain Regulate Both Innate and Conditioned Nociceptive Avoidance." Cell Rep 24(6): 1573-1584.

Huetteroth, W., E. Perisse, S. Lin, M. Klappenbach, C. Burke and S. Waddell (2015). "Sweet taste and nutrient value subdivide rewarding dopaminergic neurons in Drosophila." Curr Biol 25(6): 751-758.

Hyman, S. E., R. C. Malenka and E. J. Nestler (2006). "Neural mechanisms of addiction: the role of reward-related learning and memory." Annu Rev Neurosci 29: 565-598.

Ichinose, T., Y. Aso, N. Yamagata, A. Abe, G. M. Rubin and H. Tanimoto (2015). "Reward signal in a recurrent circuit drives appetitive longterm memory formation." Elife 4: e10719.

Imperato, A. and G. Di Chiara (1986). "Preferential stimulation of dopamine release in the nucleus accumbens of freely moving rats by ethanol." J Pharmacol Exp Ther 239(1): 219-228.

Kahsai, L. and T. Zars (2011). "Learning and memory in Drosophila: behavior, genetics, and neural systems." Int Rev Neurobiol 99: 139167.

Kaun, K. R., R. Azanchi, Z. Maung, J. Hirsh and U. Heberlein (2011). "A Drosophila model for alcohol reward." Nat Neurosci 14(5): 612-619.

Kegeles, L. S., G. Horga, R. Ghazzaoui, R. Rosengard, N. Ojeil, X. Xu, M. Slifstein, I. Petrakis, S. S. O'Malley, J. H. Krystal and A. AbiDargham (2018). "Enhanced Striatal Dopamine Release to Expectation of Alcohol: A Potential Risk Factor for Alcohol Use Disorder." Biol Psychiatry Cogn Neurosci Neuroimaging 3(7): 591598.

Keiflin, R. and P. H. Janak (2015). "Dopamine Prediction Errors in Reward Learning and Addiction: From Theory to Neural Circuitry." Neuron 88(2): 247-263.

Keleman, K., T. Micheler and V. p. members (2009.8.5). "RNAi-phiC31 construct and insertion data submitted by the Vienna Drosophila RNAi Center ".

Knudsen, E. I. (2007). "Fundamental components of attention." Annu Rev Neurosci 30: 57-78.

Kohl, J., J. Ng, S. Cachero, E. Ciabatti, M. J. Dolan, B. Sutcliffe, A. Tozer, S. Ruehle, D. Krueger, S. Frechter, T. Branco, M. Tripodi and G. S. Jefferis (2014). "Ultrafast tissue staining with chemical tags." Proc Natl Acad Sci U S A 111(36): E3805-3814.

Lewis, L. P., K. P. Siju, Y. Aso, A. B. Friedrich, A. J. Bulteel, G. M. Rubin and I. C. Grunwald Kadow (2015). "A Higher Brain Circuit for Immediate Integration of Conflicting Sensory Information in Drosophila." Curr Biol 25(17): 2203-2214.

Lima, S. Q. and G. Miesenbock (2005). "Remote control of behavior through genetically targeted photostimulation of neurons." Cell 121(1): 141-152.

Liu, C., P. Y. Placais, N. Yamagata, B. D. Pfeiffer, Y. Aso, A. B. Friedrich, I. Siwanowicz, G. M. Rubin, T. Preat and H. Tanimoto (2012). "A subset of dopamine neurons signals reward for odour memory in Drosophila." Nature 488(7412): 512-516.

Liu, G., H. Seiler, A. Wen, T. Zars, K. Ito, R. Wolf, M. Heisenberg and L. Liu (2006). "Distinct memory traces for two visual features in the Drosophila brain." Nature 439(7076): 551-556.

Masek, P., K. Worden, Y. Aso, G. M. Rubin and A. C. Keene (2015). "A dopamine-modulated neural circuit regulating aversive taste memory in Drosophila." Curr Biol 25(11): 1535-1541.

Morikawa, H. and R. A. Morrisett (2010). "Ethanol action on dopaminergic neurons in the ventral tegmental area: interaction with intrinsic ion channels and neurotransmitter inputs." Int Rev Neurobiol 91: 235288.

Nuñez, K. M., R. Azanchi and K. R. Kaun (2018). "Cue-Induced Ethanol Seeking in Drosophila melanogaster Is Dose-Dependent." Front Physiol 9: 438.

Nutt, D. J., A. Lingford-Hughes, D. Erritzoe and P. R. Stokes (2015). "The dopamine theory of addiction: 40 years of highs and lows." Nat Rev Neurosci 16(5): 305-312.

Ollion, J., J. Cochennec, F. Loll, C. Escude and T. Boudier (2013). "TANGO: a generic tool for high-throughput 3D image analysis for studying nuclear organization." Bioinformatics 29(14): 1840-1841.

Owald, D., J. Felsenberg, C. B. Talbot, G. Das, E. Perisse, W. Huetteroth and S. Waddell (2015). "Activity of defined mushroom body output neurons underlies learned olfactory behavior in Drosophila." Neuron 86(2): 417-427.

Owald, D., S. Lin and S. Waddell (2015). "Light, heat, action: neural control of fruit fly behaviour." Philos Trans R Soc Lond B Biol Sci 370(1677): 20140211.

Owald, D. and S. Waddell (2015). "Olfactory learning skews mushroom body output pathways to steer behavioral choice in Drosophila." Curr Opin Neurobiol 35: 178-184.

Perisse, E., Y. Yin, A. C. Lin, S. Lin, W. Huetteroth and S. Waddell (2013). "Different kenyon cell populations drive learned approach and avoidance in Drosophila." Neuron 79(5): 945-956.

Petruccelli, E., M. Feyder, N. Ledru, Y. Jaques, E. Anderson and K. R. Kaun (2018). "Alcohol Activates Scabrous-Notch to Influence Associated Memories." Neuron 100(5): 1209-1223 e1204.

Pfeiffer, B. D., A. Jenett, A. S. Hammonds, T. T. Ngo, S. Misra, C. Murphy, A. Scully, J. W. Carlson, K. H. Wan, T. R. Laverty, C. Mungall, R. Svirskas, J. T. Kadonaga, C. Q. Doe, M. B. Eisen, S. E. Celniker and G. M. Rubin (2008). "Tools for neuroanatomy and neurogenetics in Drosophila." Proc Natl Acad Sci U S A 105(28): 9715-9720.

Pfeiffer, B. D., J. W. Truman and G. M. Rubin (2012). "Using translational enhancers to increase transgene expression in Drosophila." Proc Natl Acad Sci U S A 109(17): 6626-6631.

Pimentel, D., J. M. Donlea, C. B. Talbot, S. M. Song, A. J. F. Thurston and G. Miesenbock (2016). "Operation of a homeostatic sleep switch." Nature 536(7616): 333-337.

Qian, Y., Y. Cao, B. Deng, G. Yang, J. Li, R. Xu, D. Zhang, J. Huang and $Y$. Rao (2017). "Sleep homeostasis regulated by $5 \mathrm{HT} 2 \mathrm{~b}$ receptor in a small subset of neurons in the dorsal fan-shaped body of drosophila." Elife 6.

Robbins, T. W. and B. J. Everitt (2002). "Limbic-striatal memory systems and drug addiction." Neurobiol Learn Mem 78(3): 625-636.

Romo, R. and W. Schultz (1990). "Dopamine neurons of the monkey midbrain: contingencies of responses to active touch during selfinitiated arm movements." J Neurophysiol 63(3): 592-606.

Roy, D. S., T. Kitamura, T. Okuyama, S. K. Ogawa, C. Sun, Y. Obata, A. Yoshiki and S. Tonegawa (2017). "Distinct Neural Circuits for the Formation and Retrieval of Episodic Memories." Cell 170(5): 10001012 e1019. 
bioRxiv preprint doi: https://doi.org/10.1101/578401; this version posted March 15,2019 . The copyright holder for this preprint (which was not certified by peer review) is the author/funder, who has granted bioRxiv a license to display the preprint in perpetuity. It is made available under aCC-BY-ND 4.0 International license.

Scaplen et al., 15 Mar 2019 - preprint copy - BioRxiv

Scaplen, K. M. and K. R. Kaun (2016). "Reward from bugs to bipeds: a comparative approach to understanding how reward circuits function." J Neurogenet 30(2): 133-148.

Scaplen, K. M., N. J. Mei, H. A. Bounds, S. L. Song, R. Azanchi and K. R. Kaun (2019). "Automated real-time quantification of group locomotor activity in Drosophila melanogaster." Scientific Reports 9(4427): 116.

Schindelin, J., I. Arganda-Carreras, E. Frise, V. Kaynig, M. Longair, T. Pietzsch, S. Preibisch, C. Rueden, S. Saalfeld, B. Schmid, J. Y. Tinevez, D. J. White, V. Hartenstein, K. Eliceiri, P. Tomancak and A. Cardona (2012). "Fiji: an open-source platform for biological-image analysis." Nat Methods 9(7): 676-682.

Schleyer, M., T. Saumweber, W. Nahrendorf, B. Fischer, D. von Alpen, D. Pauls, A. Thum and B. Gerber (2011). "A behavior-based circuit model of how outcome expectations organize learned behavior in larval Drosophila." Learn Mem 18(10): 639-653.

Schultz, W. (2007). "Multiple dopamine functions at different time courses." Annu Rev Neurosci 30: 259-288.

Schultz, W. (2015). "Neuronal Reward and Decision Signals: From Theories to Data." Physiol Rev 95(3): 853-951.

Schultz, W. (2016). "Dopamine reward prediction error coding." Dialogues Clin Neurosci 18(1): 23-32.

Sitaraman, D., Y. Aso, X. Jin, N. Chen, M. Felix, G. M. Rubin and M. N. Nitabach (2015). "Propagation of Homeostatic Sleep Signals by Segregated Synaptic Microcircuits of the Drosophila Mushroom Body." Curr Biol 25(22): 2915-2927.

Syed, E. C., L. L. Grima, P. J. Magill, R. Bogacz, P. Brown and M. E. Walton (2016). "Action initiation shapes mesolimbic dopamine encoding of future rewards." Nat Neurosci 19(1): 34-36.

Talay, M., E. B. Richman, N. J. Snell, G. G. Hartmann, J. D. Fisher, A. Sorkac, J. F. Santoyo, C. Chou-Freed, N. Nair, M. Johnson, J. R. Szymanski and G. Barnea (2017). "Transsynaptic Mapping of Second-Order Taste Neurons in Flies by trans-Tango." Neuron 96(4): 783-795 e784.

Torregrossa, M. M., P. R. Corlett and J. R. Taylor (2011). "Aberrant learning and memory in addiction." Neurobiol Learn Mem 96(4): 609-623.

Torregrossa, M. M. and J. R. Taylor (2013). "Learning to forget: manipulating extinction and reconsolidation processes to treat addiction." Psychopharmacology (Berl) 226(4): 659-672.

Trantham-Davidson, H. and L. J. Chandler (2015). "Alcohol-induced alterations in dopamine modulation of prefrontal activity." Alcohol 49(8): 773-779.

Troup, M., M. H. Yap, C. Rohrscheib, M. J. Grabowska, D. Ertekin, R. Randeniya, B. Kottler, A. Larkin, K. Munro, P. J. Shaw and B. van Swinderen (2018). "Acute control of the sleep switch in Drosophila reveals a role for gap junctions in regulating behavioral responsiveness." Elife 7.

Volkow, N. D. and M. Morales (2015). "The Brain on Drugs: From Reward to Addiction." Cell 162(4): 712-725.

Waddell, S. (2010). "Dopamine reveals neural circuit mechanisms of fly memory." Trends Neurosci 33(10): 457-464.

Waddell, S. (2013). "Reinforcement signalling in Drosophila; dopamine does it all after all." Curr Opin Neurobiol 23(3): 324-329.

Wanat, M. J., I. Willuhn, J. J. Clark and P. E. Phillips (2009). "Phasic dopamine release in appetitive behaviors and drug addiction." Curr Drug Abuse Rev 2(2): 195-213.

Wang, W., M. Darvas, G. P. Storey, I. J. Bamford, J. T. Gibbs, R. D. Palmiter and N. S. Bamford (2013). "Acetylcholine encodes longlasting presynaptic plasticity at glutamatergic synapses in the dorsal striatum after repeated amphetamine exposure." J Neurosci 33(25): 10405-10426.
Weir, P. T. and M. H. Dickinson (2015). "Functional divisions for visual processing in the central brain of flying Drosophila." Proc Natl Acad Sci U S A 112(40): E5523-5532.

Weir, P. T., B. Schnell and M. H. Dickinson (2014). "Central complex neurons exhibit behaviorally gated responses to visual motion in Drosophila." J Neurophysiol 111(1): 62-71.

Wolff, T., N. A. Iyer and G. M. Rubin (2015). "Neuroarchitecture and neuroanatomy of the Drosophila central complex: A GAL4-based dissection of protocerebral bridge neurons and circuits." J Comp Neurol 523(7): 997-1037.

Yamagata, N., M. Hiroi, S. Kondo, A. Abe and H. Tanimoto (2016). "Suppression of Dopamine Neurons Mediates Reward." PLoS Biol 14(12): e1002586.

Yamagata, N., T. Ichinose, Y. Aso, P. Y. Placais, A. B. Friedrich, R. J. Sima, T. Preat, G. M. Rubin and H. Tanimoto (2015). "Distinct dopamine neurons mediate reward signals for short- and long-term memories." Proc Natl Acad Sci U S A 112(2): 578-583.

Yoshimoto, K., W. J. McBride, L. Lumeng and T. K. Li (1992). "Alcohol stimulates the release of dopamine and serotonin in the nucleus accumbens." Alcohol 9(1): 17-22.

Yoshimoto, K., S. Ueda, B. Kato, Y. Takeuchi, Y. Kawai, K. Noritake and M. Yasuhara (2000). "Alcohol enhances characteristic releases of dopamine and serotonin in the central nucleus of the amygdala." Neurochem Int 37(4): 369-376. 
bioRxiv preprint doi: https://doi.org/10.1101/578401; this version posted March 15,2019 . The copyright holder for this preprint (which was not certified by peer review) is the author/funder, who has granted bioRxiv a license to display the preprint in perpetuity. It is made available under aCC-BY-ND 4.0 International license.

Scaplen et al., 15 Mar 2019 - preprint copy - BioRxiv

\begin{tabular}{|c|c|c|c|}
\hline Drosophila Strain & Expression Pattern & Source & Identifier \\
\hline$y w$ & N/A & (Pfeiffer, Jenett et al. 2008) & \\
\hline shibire $^{t s 1}$ & N/A & (Pfeiffer, Truman et al. 2012) & \\
\hline GCaMP6m & $\mathrm{N} / \mathrm{A}$ & & BDSC 42750 \\
\hline UAS-Dop2R-RNAi & dsRNA-GD11471 & (Dietzl, Chen et al. 2007) & VDRC ID: 11471 \\
\hline UAS-Dop1R1-RNAi & dsRNA-KK107258 & $\begin{array}{l}\text { (Keleman, Micheler et al. } \\
\text { 2009.8.5) }\end{array}$ & $\begin{array}{l}\text { VDRC ID: } 107258 \\
\text { (currently unavailable) } \\
\text { Flybase: FBsf0000090794 }\end{array}$ \\
\hline UAS-Dop1R2-RNAi & dsRNA-GD3391 & (Dietzl, Chen et al. 2007) & $\begin{array}{l}\text { VDRC ID: } 3391 \\
\text { (no longer available) } \\
\text { Flybase: FBsf0000073893 }\end{array}$ \\
\hline $\begin{array}{lr}\text { trans-Tango: } & \text { UAS- } \\
\text { myrGFP, } & \text { QUAS } \\
\text { mtdTomato } & (3 \times H A) ; \\
\text { brp-SNAP } & \end{array}$ & $\mathrm{N} / \mathrm{A}$ & $\begin{array}{l}\text { (Kohl, } \mathrm{Ng} \text { et al. 2014, Talay, } \\
\text { Richman et al. 2017) }\end{array}$ & \\
\hline R58E02-Gal4 & $\begin{array}{l}\text { PAM dopamine } \\
\text { neurons }\end{array}$ & (Liu, Placais et al. 2012) & BDSC_41347 \\
\hline R58E02; mcD8::GFP & $\begin{array}{l}\text { PAM dopamine } \\
\text { neurons }\end{array}$ & & $\begin{array}{l}\text { BDSC_79626, } \\
\text { BDSC_41347 }\end{array}$ \\
\hline HL9-Gal4 & $\begin{array}{l}\text { PAM dopamine } \\
\text { neurons }\end{array}$ & $\begin{array}{l}\text { (Claridge-Chang, Roorda et al. } \\
\text { 2009) }\end{array}$ & Flybase: FBtp0073020 \\
\hline MB058B-split-Gal4 & PPL1 - $\alpha 2 \alpha^{\prime} 2(5)$ & (Aso, Hattori et al. 2014) & FlyLight Robot ID: 2135107 \\
\hline MB109B-split-Gal4 & $\begin{array}{l}\text { PAM } \beta^{`} 2 a(5), \gamma 5(1) \\
\text { PAM } \beta^{\prime} 2 a(5), \gamma 5(1)\end{array}$ & (Aso, Hattori et al. 2014) & FlyLight Robot ID: 2135157 \\
\hline MB040B-split-Gal4 & $\begin{array}{l}\text { PAM- } \alpha 1(2), \beta^{\prime} 1 \mathrm{ap}(1), \\
\beta^{\prime} 1 \mathrm{~m}(1), \\
\beta^{\prime} 2 \mathrm{a}(1), \beta^{\prime} 2 \mathrm{~m}(2), \\
\beta^{\prime} 2 \mathrm{p}(1), \beta^{\prime} 1(1), \beta 2(3), \\
\gamma^{3}(3), \gamma 4(3), \gamma 5(3)\end{array}$ & (Aso, Hattori et al. 2014) & N/A \\
\hline MB042B-split-Gal4 & $\begin{array}{l}\text { PAM- } \alpha 1(1), \beta^{\prime} 1 \mathrm{ap}(1), \\
\beta^{\prime} 1 \mathrm{~m}(1), \beta^{\prime} 2 \mathrm{~m}(2), \\
\beta^{\prime} 2 \mathrm{p}(1), \beta^{\prime} 1(1), \gamma 3(3), \\
\gamma 4(3), \gamma 5<\gamma 1 \gamma 2(1), \gamma 5(3)\end{array}$ & (Aso, Hattori et al. 2014) & FlyLight Robot ID: 2135092 \\
\hline MB188B-split-Gal4 & $\begin{array}{l}\text { PAM- } \beta \text { 1ap(5), } \\
\beta^{`} 1 \mathrm{~m}(5), \gamma 3(3), \gamma 4(2)\end{array}$ & (Aso, Hattori et al. 2014) & FlyLight Robot ID: 2135236 \\
\hline MB032B-split-Gal4 & $\begin{array}{l}\text { PAM- } \beta^{\wedge} 2 m(4), \beta^{2} 2 p(1), \\
\beta 2 \beta^{\prime} 2 a(1), \gamma 3(1)\end{array}$ & (Aso, Hattori et al. 2014) & FlyLight Robot ID: 2135083 \\
\hline MB087C-split-Gal4 & PAM- $\beta 22$ (a(1) & (Aso, Hattori et al. 2014) & FlyLight Robot ID: 2135135 \\
\hline MB301B-split-Gal4 & $\begin{array}{l}\text { PAM- } \beta^{`} 2 \mathrm{~m}(1) \text {, } \\
\beta 2 \beta^{`} 2 \mathrm{a}(3)\end{array}$ & (Aso, Hattori et al. 2014) & FlyLight Robot ID: 2135349 \\
\hline MB002B-split-Gal4 & $\begin{array}{l}\text { MBON- } \beta^{\prime} 2 \mathrm{mp}(4), \\
\gamma 5 \beta^{\prime} 2 \mathrm{a}(2)\end{array}$ & (Aso, Hattori et al. 2014) & FlyLight Robot ID:2135053 \\
\hline MB011B-split-Gal4 & $\begin{array}{l}\text { MBON- } \gamma 5 \beta^{\prime} 2 \mathrm{a}(4), \\
\beta^{\prime} 2 \mathrm{mp}(3), \\
\beta^{\prime} 2 \mathrm{mp} \text { bilateral (3) }\end{array}$ & (Aso, Hattori et al. 2014) & FlyLight Robot ID:2135062 \\
\hline MB-210B-split-Gal4 & $\begin{array}{l}\text { MBON- } \gamma 5 \beta^{\prime} 2 a(5), \\
\beta^{\prime} 2 m p(4), \\
\beta^{\prime} 2 m p \_ \text {bilateral (1) }\end{array}$ & (Aso, Hattori et al. 2014) & FlyLight Robot ID: 2135258 \\
\hline MB018B-split-Gal4 & MBON- $\alpha 2(4)$ & (Aso, Hattori et al. 2014) & FlyLight Robot ID: 2135069 \\
\hline MB399B-split-Gal4 & MBON- $\beta 2 \beta 2 a(2)$ & (Aso, Hattori et al. 2014) & FlyLight Robot ID: 2501738 \\
\hline MB074C-split-Gal4 & $\begin{array}{l}\text { MBON-- } \gamma 5 \beta^{`} 2 \mathrm{a}(1) \\
\beta^{`} 2 \mathrm{mp}(4), \beta 2 \beta^{\prime} 2 \mathrm{a}(3)\end{array}$ & (Aso, Hattori et al. 2014) & FlyLight Robot ID: 2135112 \\
\hline
\end{tabular}

Table 1. Fly Strains used in this manuscript. 